\title{
Evolution and rotation of large-scale photospheric magnetic fields of the Sun during cycles 21-23
}

\section{Periodicities, north-south asymmetries and r-mode signatures ${ }^{\star}$}

\author{
R. Knaack ${ }^{1}$, J. O. Stenflo ${ }^{1,2}$, and S. V. Berdyugina ${ }^{1,3}$ \\ 1 Institute of Astronomy, ETH-Zentrum, 8092 Zurich, Switzerland \\ e-mail: [rknaack;stenflo; sveta]@astro.phys.ethz.ch \\ ${ }^{2}$ Faculty of Mathematics and Science, University of Zurich, 8057 Zurich, Switzerland \\ 3 Astronomy Division, PO Box 3000, 90014 University of Oulu, Finland
}

Received 29 September 2004 / Accepted 24 March 2005

\begin{abstract}
We present the results of an extensive time series analysis of longitudinally-averaged synoptic maps, recorded at the National Solar Observatory (NSO/Kitt Peak) from 1975 to 2003, and provide evidence for a multitude of quasi-periodic oscillations in the photospheric magnetic field of the Sun. In the low frequency range, we have located the sources of the $3.6 \mathrm{yr}$, $1.8 \mathrm{yr}$, and $1.5 \mathrm{yr}$ periodicities that were previously detected in the north-south asymmetry of the unsigned photospheric flux (Knaack et al. 2004, A\&A, 418, L17). In addition, quasi-periodicities around $2.6 \mathrm{yr}$ and $1.3 \mathrm{yr}$ have been found. The $1.3 \mathrm{yr}$ period is most likely related to large-scale magnetic surges toward the poles and appeared in both hemispheres at intermediate latitudes $\sim 30^{\circ}-55^{\circ}$ during the maxima of all three cycles $21-23$, being particularly pronounced during cycle 22 . Periods near $1.3 \mathrm{yr}$ have recently been reported in the rotation rate at the base of the convection zone (Howe et al. 2000, Science, 287, 2456), in the interplanetary magnetic field and geomagnetic activity (Lockwood 2001, J. Geophys. Res., 106, 16021) and in sunspot data (Krivova \& Solanki 2002, A\&A, 394, 701). In the intermediate frequency range, we have found a series of quasiperiodicities of 349-307 d, $282 \pm 4$ d, 249-232 d, 222-209 d, $177 \pm 2$ d, 158-151 d, 129-124 d and 103-100 d, which are in good agreement with period estimates for Rossby-type waves and occurred predominantly in the southern hemisphere. We provide evidence that the best known of these periodicities, the Rieger period around $155 \mathrm{~d}$, appeared in the magnetic flux not only during cycle 21 but also during cycle 22 , likely even during cycle 23 . The high frequency range, which covers the solar rotation periods, shows a dominant (synodic) $28.1 \pm 0.1 \mathrm{~d}$ periodicity in the southern hemisphere during cycles 21 and 22 . A periodicity around 25.0-25.5 d occurred in the south during all three cycles. The large-scale magnetic field of the northern hemisphere showed dominant rotation periods at $26.9 \pm 0.1 \mathrm{~d}$ during cycle 21 , at $28.3-29.0 \mathrm{~d}$ during cycle 22 and at $26.4 \pm 0.1 \mathrm{~d}$ during cycle 23 .
\end{abstract}

Key words. Sun: photosphere - Sun: magnetic fields - Sun: sunspots - Sun: activity

\section{Introduction}

The Sun's magnetic activity appears to exhibit both periodic and quasi-periodic variations on different time scales. In particular the explanation of the basic mechanisms underlying the well-known Hale magnetic cycle with an average period of 22 years and the sunspot cycle with half this period remains one of the fundamental challenges in solar physics. The cyclic behavior of the solar large-scale magnetic field has, however, provided invaluable information about the physical processes involved and therefore the interest in finding additional periods is large. Since the discovery of the Rieger period around 155 days in the occurrence of high-energetic flares

* Figures 8-10 and Tables 4-6 are only available in electronic form at http://www.edpsciences.org
(Rieger et al. 1984; Kiplinger et al. 1984; Bogart \& Bai 1985), extensive investigations have been carried out to search for intermediate periodicities in the range between 27 days and 11 years in various solar activity indices (cf. Bai 2003b, and references therein).

Recently, we have analyzed the north-south asymmetry of the photospheric magnetic flux from 1975 to 2003 and found three pronounced quasi-periodicities (Knaack et al. 2004, henceforth referred to as Paper I). The first periodicity of $1.50 \pm$ $0.04 \mathrm{yr}$ occurred during $1978-1984$ and was accompanied by a gradual shift in the excess of magnetic flux from the northern to the southern hemisphere. The south remained dominant during the following interval 1984-1995, and the southern flux excess varied with a $3.6 \pm 0.3 \mathrm{yr}$ periodicity. Finally, the flux excess shifted back to the north during 1995-2001, modulated with a period of $1.79 \pm 0.06 \mathrm{yr}$. 

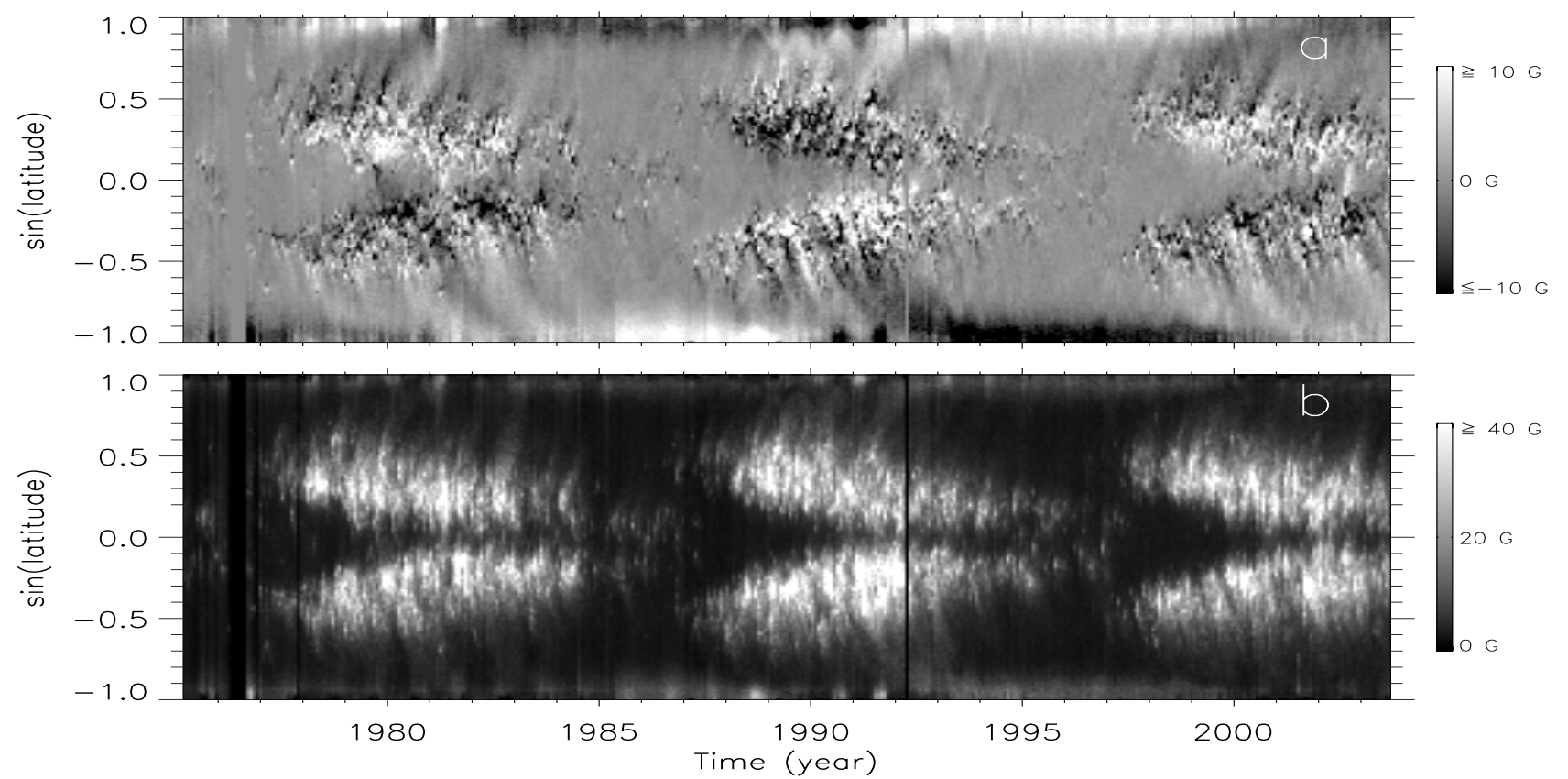

Fig. 1. a) Butterfly diagram of the net (signed) magnetic flux density, averaged over longitude for each Carrington rotation. Sine latitude ranges from -1 (south pole) to +1 (north pole). Positive polarities appear white, negative polarities black, as defined by the color bar. For better contrast, the upper and lower cut offs have been set to \pm 10 Gauss. The actual maximum is $41 \mathrm{G}$, the minimum $-29 \mathrm{G}$. b) The same for the total (unsigned) magnetic flux density (i.e. the average of the absolute values). The maximum is $82 \mathrm{G}$.

Asymmetries between the northern and the southern hemisphere were previously found in various solar indices, for instance in the distribution of flares (Reid 1968; Roy 1977; Ichimoto et al. 1985; Verma 1987; Bai 1990; Garcia 1990; Atac \& Ozguc 1996; Li et al. 1998; Temmer et al. 2001; Bai 2003b; Joshi \& Joshi 2004) and filaments (Hansen \& Hansen 1975; Vizoso \& Ballester 1987; Duchlev \& Dermendjiev 1996; Duchlev 2001), in the photospheric magnetic flux (Howard 1974; Mouradian \& Soru-Escaut 1991; Knaack et al. 2004), in the rotation velocities of photospheric magnetic fields (Antonucci et al. 1990; Javaraiah \& Gokhale 1997), and in the relative sunspot numbers and sunspot areas (Newton \& Milsom 1955; Waldmeier 1971; Swinson et al. 1986; Vizoso \& Ballester 1989, 1990; Carbonell et al. 1993; Oliver \& Ballester 1994; Verma 2000; Li et al. 2002; Temmer et al. 2002; Vernova et al. 2002; Knaack et al. 2004). North-south asymmetries may be due to phase differences between the magnetic activity in both hemispheres (Waldmeier 1971; Swinson et al. 1986). The interference between the dominant dipolar mode and modes with quadrupolar symmetry with respect to the equator (which can be excited through nonlinear or stochastic effects) may strongly enhance the north-south asymmetry between the hemispheres (Ossendrijver 2003). For instance, the few sunspots observed during the Maunder minimum $(\sim 1660-1720)$ were mainly concentrated in the southern hemisphere (Sokoloff \& Nesme-Ribes 1994).

The aim of the present analysis is to verify the existence and locate the sources of the $1.5 \mathrm{yr}, 1.8 \mathrm{yr}$, and $3.6 \mathrm{yr}$ periodicities reported in Paper I. Moreover, we have searched for any additional periodic or quasi-periodic variations that may have occurred in the large-scale magnetic field of the Sun during the last three cycles 21-23. For this purpose, we have used the same data set as in Paper I, namely the synoptic Carrington rotation maps of the photospheric magnetic field recorded at the National Solar Observatory at Kitt Peak (NSO/KP) from 1975 to 2003, and applied a technique introduced by Hoeksema \& Scherrer (1987) and Antonucci et al. (1990). The underlying notion is to compile a latitude-time diagram of the magnetic flux, also known as "butterfly" diagram (cf. Fig. 1), and to compute the power spectra of the time series in each latitude zone. The spectral power is then plotted in latitude-frequency contour maps, which readily allow the identification of the latitude and the frequency of (quasi-) periodic signals. We have extended the original method by applying wavelet analysis, which additionally yields the time interval during which an oscillation occurred.

The following frequency intervals in the net (signed) as well as the total (unsigned) flux have been examined for cycles 21-23: "low" frequencies in the range $1.1 \mathrm{nHz}-30 \mathrm{nHz}$ (corresponding to periods of $29 \mathrm{yr}-1 \mathrm{yr}$ ), "intermediate" frequencies in the range $30 \mathrm{nHz}-120 \mathrm{nHz}(386 \mathrm{~d}-96 \mathrm{~d})$, and "high" frequencies in the ranges $390 \mathrm{nHz}-470 \mathrm{nHz}$ $(29.7 \mathrm{~d}-24.6 \mathrm{~d})$ and $780 \mathrm{nHz}-940 \mathrm{nHz}(14.8 \mathrm{~d}-12.3 \mathrm{~d})$. Data and methods are described in more detail in Sect. 2.

In Sect. 3, the results for the low frequency range are presented. We have found evidence in the unsigned flux that the $1.5 \mathrm{yr}$ and the $3.6 \mathrm{yr}$ periods were present in both hemispheres, while the $1.8 \mathrm{yr}$ period occurred only in the south. In addition, a quasi-biennial oscillation (Obridko \& Shelting 2001) around $2.6 \mathrm{yr}$ was detected, and an intermittent $1.30 \pm 0.03 \mathrm{yr}$ periodicity occurred in the unsigned flux at intermediate latitudes in both hemispheres during the maxima of all three cycles. The corresponding periodicities in the signed flux are slightly larger, $1.50 \pm 0.04 \mathrm{yr}$ in the north and $1.36 \pm 0.04 \mathrm{yr}$ in the 
south. Oscillations at $1.3 \mathrm{yr}$ have recently been observed in the rotation rate at the base of the convection zone (Howe et al. 2000), in the heliosphere (Lockwood 2001, and references therein) and in sunspot areas and sunspot numbers (Krivova \& Solanki 2002), but not yet directly in the large-scale photospheric magnetic field. We assume that the $\sim 1.3 \mathrm{yr}$ period in the photosphere is caused by large-scale magnetic surges toward the poles (Wang et al. 1989), which are nicely seen in Fig. 1a, particularly during the interval 1989-1993. We will discuss this point in more detail in Sect. 3.1.1.

In Sect. 4 , the results for the intermediate frequency range are shown. We have found a series of significant peaks, most of them confined to the narrow latitude band $\sim 10^{\circ} \mathrm{S}-20^{\circ} \mathrm{S}$. This might be related to the circumstance that the southern hemisphere dominated during cycle 22, while the two hemispheres were approximately balanced during cycles 21 and 23 (cf. Paper I). In the unsigned flux, the peaks occur at $33.2-37.7 \mathrm{nHz}$ $(349-307 \mathrm{~d}), 41.0 \pm 0.6 \mathrm{nHz}(282 \pm 4 \mathrm{~d}), 46.5-49.9 \mathrm{nHz}$ $(249-232 \mathrm{~d}), \quad 52.1-55.4 \mathrm{nHz} \quad(222-209$ d), $65.4 \quad \pm$ $0.6 \mathrm{nHz}(177 \pm 2 \mathrm{~d}), 73.1-76.5 \mathrm{nHz}(158-151 \mathrm{~d})$ and $89.7-93.1 \mathrm{nHz}(129-124 \mathrm{~d})$. Most of them are double peaks that show a frequency separation of $\Delta v \approx 3.4 \mathrm{nHz}(9.3 \mathrm{yr})$, which is close to the $\sim 11 \mathrm{yr}$ sunspot cycle. The results for the signed flux are similar but certain discrepancies exist. For instance, an additional double peak at $111.9-115.2 \mathrm{nHz}$ (103-100 d) occurs only in the signed flux. Although most of these periodicities were reported earlier in several solar activity indicators (e.g. Wolff 1983; Rieger et al. 1984; Delache et al. 1985; Lean \& Brueckner 1989; Pap et al. 1990; Bai \& Sturrock 1991; Bai 2003b; Lou et al. 2003), it is the first time that they have collectively been detected in direct observations of the photospheric magnetic field. The Rieger period around $155 \mathrm{~d}$ is of special interest and will be discussed in detail in Sect. 4.1, together with the similar "Rieger-type" periodicities (periods close to integer multiples of 25.5 days, cf. Bai $\&$ Sturrock 1991; Lou 2000) in Sect. 4.2. There have been suggestions (Sturrock et al. 1999; Lou 2000; Sturrock 2004) that the Rieger and Rieger-type periodicities may be related to physical properties of Rossby-type waves, often denoted as $r$-modes (cf. Sect. 6). We have compared the detected intermediate quasi-periodicities in the photospheric magnetic flux with period estimates for Rossby-type waves and found that they are generally in good agreement.

In Sect. 5, the results for the high frequency range are discussed. We have analyzed the time series as a whole and also for the separate cycles 21 (1976.5-1986.8), 22 (1986.8-1996.7) and 23 (data available from 1996.7 to 2003.7). The results for cycle 21 are in good agreement with the conclusions of Antonucci et al. (1990) that the large-scale field rotated more rapidly in the northern than in the southern hemisphere. The dominant synodic rotation frequency in the south was $412 \pm 1.5 \mathrm{nHz}(28.1 \pm 0.1 \mathrm{~d})$ and in the north $431 \pm$ $1.5 \mathrm{nHz}(26.9 \pm 0.1 \mathrm{~d})$. Significant power at these two frequencies extended over a large latitudinal range. During cycle 22 , the $28.1 \mathrm{~d}$ period became even more distinct in the south. In the north, there was significant power around $27.3 \pm 0.1 \mathrm{~d}$ but most power occurred around periods of 28.3-28.6 d in the signed flux and around $29.0 \pm 0.1 \mathrm{~d}$ in the unsigned flux, respectively. During the current cycle 23 , the $28.1 \mathrm{~d}$ period has almost vanished in the south. Instead, most power appears in the range of 26.7-27.3 d. In the north, a dominant peak is seen at $26.4 \pm 0.1 \mathrm{~d}$. Surprisingly, a synodic period around 24.9-25.5 d appeared in the unsigned magnetic flux at latitudes $\sim 12^{\circ} \mathrm{S}-19^{\circ} \mathrm{S}$ during all three cycles, which is possibly related to the "fundamental period" proposed by Bai \& Sturrock (1991).

In Sect. 6, we briefly introduce $r$-modes and give a list of period estimates. The agreement of the detected quasiperiodicities with these period estimates supports the view that Rossby-type waves may be a viable explanation for quasiperiodic oscillations in the photospheric magnetic flux. Finally, the conclusions are presented in Sect. 7.

\section{Data and reduction procedure}

The NSO/Kitt Peak Synoptic maps of the photospheric magnetic field were constructed from daily full-disk magnetograms spanning a full Carrington rotation $(1 \mathrm{CR}=27.2753 \mathrm{~d})$. These maps approximate the magnetic flux density $B(\varphi, \sin \vartheta)$ in the photosphere as a function of heliographic longitude $\varphi\left(0^{\circ} \leqq\right.$ $\left.\varphi<360^{\circ}\right)$ and sine latitude $\vartheta(-1<\sin \vartheta<+1)$ under the assumption that the magnetic fields are vertical (e.g. Worden \& Harvey 2000, and references therein). The entire data set consists of 377 synoptic maps and covers Carrington rotations 1625-2007 (February 1975-August 2003). Data gaps are rotations 1640-1644 and 1854. The resolution of each map is $360 \times 180$ pixels, i.e. $1^{\circ}$ in longitude and 0.011 in sine latitude.

\subsection{Butterfly diagrams}

Butterfly (or latitude-time) diagrams of the net (signed) and the total (unsigned) magnetic flux are shown in Figs. 1a and 1b, respectively ${ }^{1}$. They were calculated by averaging either the signed or the unsigned flux values of each synoptic map over all longitudes $1^{\circ}-360^{\circ}$. Missing data were replaced by zero. Thus, each row of the two butterfly diagrams shows the time variation of the net or total magnetic flux at a specific (sine) latitude. Each time series has 383 data points, the time step is $\Delta t=1 \mathrm{CR}$. This time step can be varied depending on the choice of the longitude bins over which the averaging is done. Averaging $B(\varphi, \sin \vartheta)$ (or its absolute value) over the 10 longitude bins $1^{\circ}-36^{\circ}, 37^{\circ}-72^{\circ}, \ldots, 325^{\circ}-360^{\circ}$ (instead of $1^{\circ}-360^{\circ}$ as in Fig. 1) increases the number of data points in each time series by a factor of 10 and reduces the time step to $\Delta t=\frac{1}{10} \mathrm{CR}=2.72753 \mathrm{~d}$. Thus we used these finer sampled time series to investigate not only long-term variations but also rotational effects of the magnetic flux.

\subsection{Time series analysis}

Fourier power spectra (FPS) were calculated for each time series of the 180 latitude zones of the butterfly diagrams using the Fast Fourier Transform (FFT). The spectral power was then

\footnotetext{
1 For convenience, numerical values are given in average flux density units of Gauss $\left(10^{-4} \mathrm{Vsm}^{-2}\right)$. To convert to Weber (1 Vs), multiply by $3.42 \times 10^{12}$.
} 
plotted in latitude-frequency diagrams (Hoeksema \& Scherrer 1987; Antonucci et al. 1990). Periodic signals in the time series hence show up as regions of increased power in these diagrams and the latitudes and frequencies of the power peaks can be readily determined. However, the lifetime of an oscillation may be limited and no information is obtained about when the quasi-periodic signal occurred in the time interval 1975-2004.

This problem can be dealt with in two ways. Firstly, we used wavelet analysis to decompose a one-dimensional time series into the two-dimensional time-frequency space. Thus it is possible to determine not only the periodicities of the dominant modes of variability but also how these modes vary in time (Torrence \& Compo 1998). The resolution in time and frequency has to be chosen carefully. A good temporal resolution is required to localize the power maxima in time, whereas a good frequency resolution is necessary to determine the corresponding frequencies (see, for example, De Moortel et al. 2004). Secondly, we divided the time series into subintervals and calculated FFT latitude-frequency diagrams for each cycle 21, 22, and 23. However, since the frequency resolution decreases for short time series, low-frequency signals are poorly determined and we used this method only for high-frequency signals such as those caused by the differential rotation of the Sun. In conclusion, the combined use of latitude-frequency diagrams and wavelet analysis provided three pieces of information: the latitude, at which an oscillation occurred, its frequency (or period), and the time interval, over which the oscillation was present.

Significance levels for the FPS were calculated following the method given by Torrence \& Compo (1998). We define, for a given sine latitude, the power spectral density $P$ of the time series of the (total or net) magnetic flux density $B(\sin \vartheta, t)$ as

$P(\sin \vartheta, v) \equiv 2|\tilde{B}(\sin \vartheta, v)|^{2}, \quad 0 \leq v \leq v_{\mathrm{c}}$,

where $v$ denotes frequency, $\tilde{B}$ the Fourier transform of $B$, and $v_{\mathrm{c}}=1 /(2 \Delta t)$ the Nyquist critical frequency. Note that $\tilde{B}$ should be normalized so that the integrated power spectrum $\int_{0}^{v_{\mathrm{c}}} P \mathrm{~d} v$ equals the variance $\sigma^{2}$ of the time series (Parseval's theorem).

If we assume a Gaussian distribution for each point of the time series (i.e. white noise), then each point of the FPS should have a $\chi^{2}$ distribution with two degrees of freedom (DOF). For a given significance level $p(0<p<1)$, we can define the corresponding power

$P_{p} \equiv \frac{\sigma^{2} \chi^{2}(1-p \text {, d.o.f. })}{0.5 N \text { d.o.f. }}$

Here $N$ is the number of points in the time series and $N / 2$ the number of frequencies in the FPS (cf. Eq. (17) in Torrence \& Compo 1998). Note that $P_{p}$ depends on $\sin \vartheta$ but not on $v$. If one were to choose randomly 100 frequencies in the interval $\left[0, v_{\mathrm{c}}\right]$, then only 1 of these frequencies would be expected to have power greater than $P_{p=0.99}$. In our case, we have $N=3830$ data points in each time series. Therefore, we would expect 2 frequencies in the FPS to randomly have power greater than the $99.9 \%$ significance level $(0.001 * N / 2=2)$, but none should exceed the $99.99 \%$ significance level by chance. This means that if we find frequencies with power above the
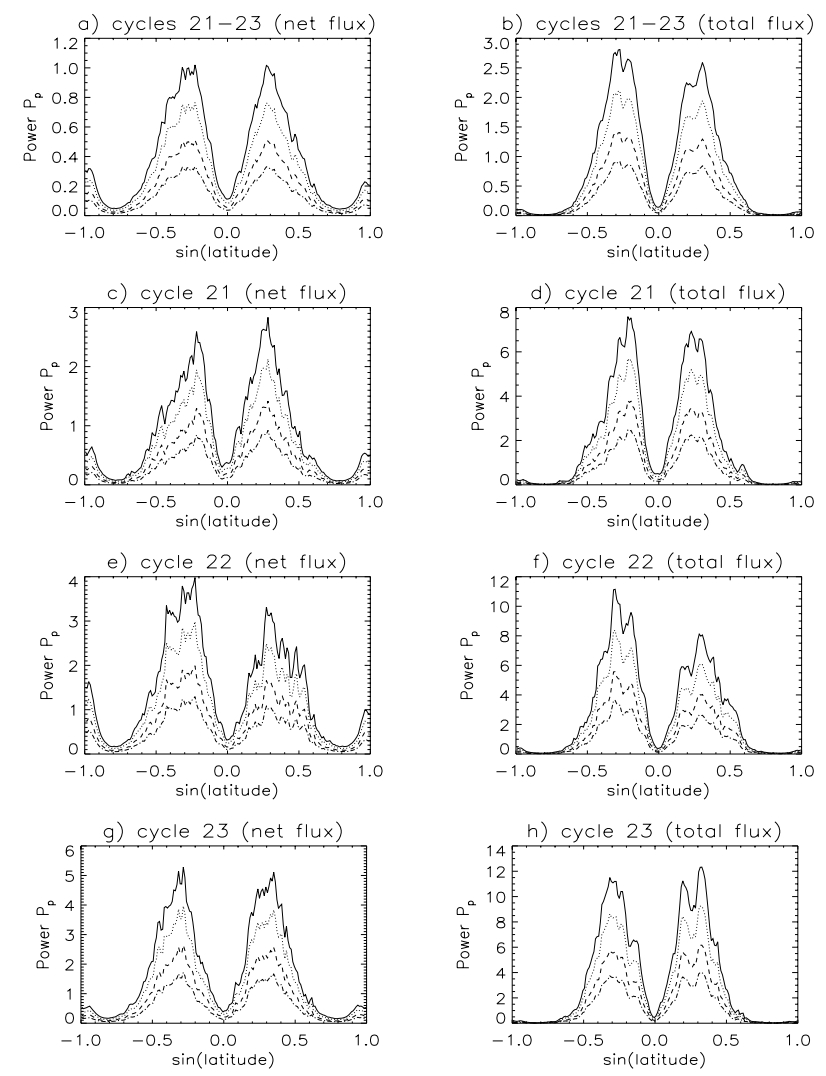

Fig. 2. Power level $P_{p}$ vs. $\sin \vartheta$ for significance levels $p=0.9999$ (solid line), $p=0.999$ (dotted), $p=0.99$ (dashed), and $p=0.95$ (dash-dotted). a) $P_{p}$ for the net magnetic flux and the entire time series (cycles $21-23, N=3830)$. b) The same for the total magnetic flux. c) and d) Cycle $21(N=1380)$. e) and f) Cycle $22(N=1320)$. g) and h) Cycle $23(N=940)$.

99.99\% significance level, we can assume that these power peaks are not just due to random noise. Figure 2 shows $P_{p}$ in arbitrary (but constant) units for the net and total magnetic flux as function of $\sin \vartheta$ for for the different cycles and for $p=0.9999$, $p=0.999, p=0.99$, and $p=0.95$. Large values of $P_{p}$ correspond to large $\sigma^{2}$. Interestingly, $P_{p}$ shows a rather distinct north-south asymmetry for cycle 22 (Figs. $2 \mathrm{e}$ and 2f), indicating a more active southern hemisphere. Cycles 21 and 23 do not show a similar asymmetry, which is in agreement with our results in Paper I. In the following sections, we will define certain regions in the latitude-frequency diagrams where the power exceeds a given significance level $p$, and denote the maximum power within these regions as $P_{\max }$.

The significance levels for the wavelet power spectra (WPS) were similarly calculated assuming a mean background spectrum modeled with a univariate lag-1 autoregressive process (for details see Torrence \& Compo 1998). Empirically, a signal which has $99.99 \%$ significance in the FPS usually has 99.0-99.9\% significance in the WPS. However, if a signal is relatively weak and intermittent, it may occur only in the WPS but not in the FPS. Thus, the latitude-frequency diagrams calculated with the FFT provide a good and efficient overview while the wavelet power spectra are in principal better suited to detect weak signals. 


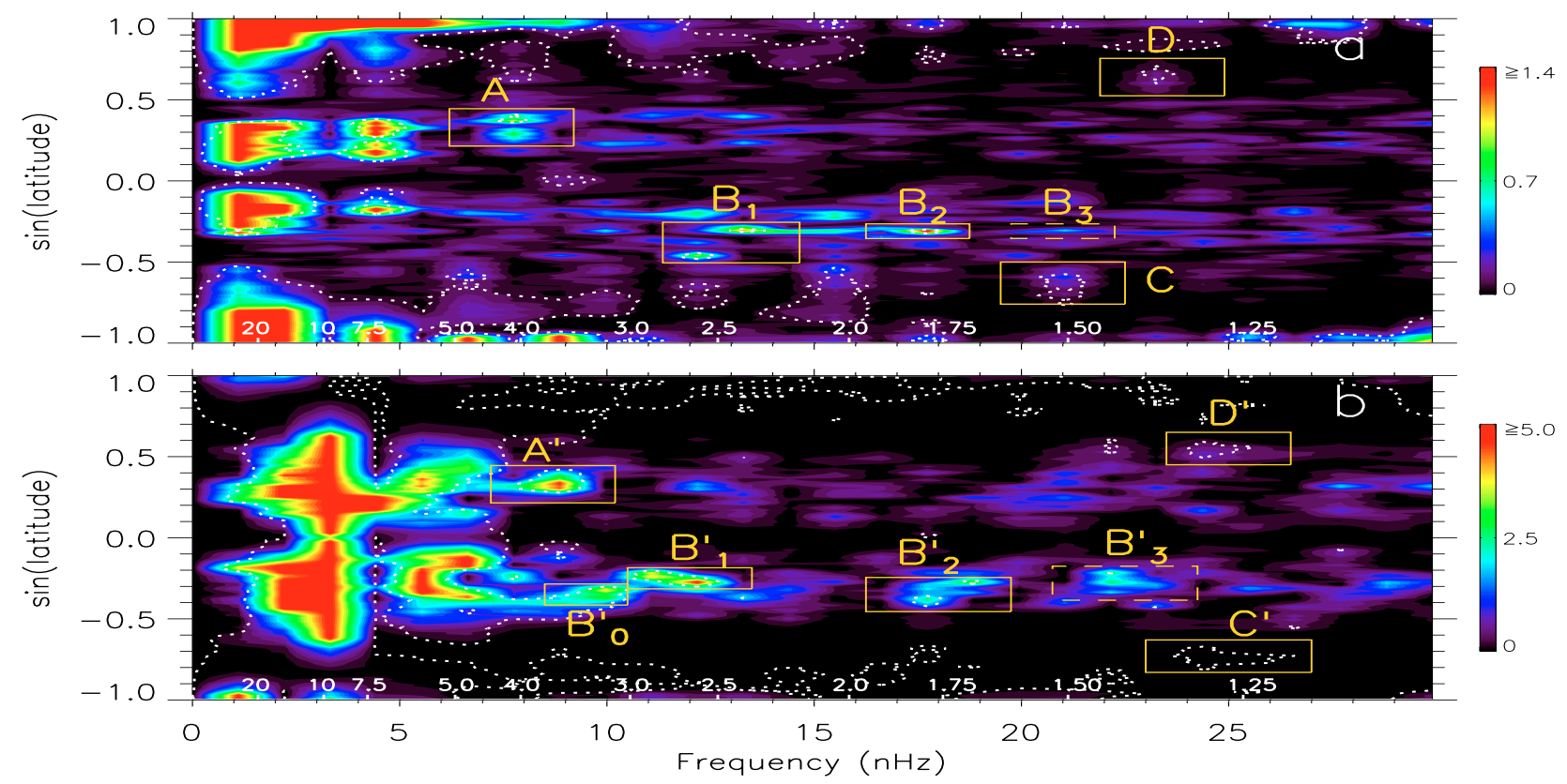

Fig. 3. a) FFT power density (in arbitrary but constant units) of the net (signed) magnetic flux vs. sine latitude and frequency. The frequency range is $1.1-29.9 \mathrm{nHz}$ (corresponding to periods $29-1 \mathrm{yr}$, indicated in white on the horizontal axis). Dotted contour lines represent the $99.99 \%$ significance level, the labeled regions A-D are listed in Table 1. The color table has been normalized to the maximum power in the range $10-30 \mathrm{nHz}$ (see the color bar). b) The same for the total (unsigned) magnetic flux.

The time interval over which a specific signal occurred (i.e. its lifetime) was determined as follows: first, the latitudes were identified where the power peak in the latitude-frequency diagram exceeded a given significance level according to Eq. (2) (usually $99.99 \%$ for the low and high frequency ranges, and 99.0-99.9\% for intermediate frequencies). Second, for each of these latitudes the WPS of the time series was calculated using Morlet wavelets with a preferably small nondimensional frequency. A Morlet wavelet is a plane wave modulated by a Gaussian and the nondimensional frequency $\omega_{0}$ (often taken to be 6, cf. Torrence \& Compo 1998) defines the number of oscillations within the wavelet itself. Increasing the nondimensional frequency increases the frequency resolution but decreases the time resolution (cf. De Moortel et al. 2004). We used the nondimensional frequencies $\omega_{0}=6$ or 9 for the low, $\omega_{0}=12$ for the intermediate and $\omega_{0}=18$ for the high frequency range. Third, the wavelet power spectrum that showed the strongest power for the considered periodicity was chosen and the boundaries of the $99 \%$ or $99.9 \%$ significance contour were used for the upper and lower limit of the lifetime of the periodicity. Please note that edge effects (i.e. the "cone of influence", cf. Torrence \& Compo 1998) were not taken into account. The general advantage of this technique is that the periodicities obtained through Fourier analysis can be cross-checked. However, it is quite extensive and not always completely unambiguous. The tabulated time intervals should therefore be seen as informative estimates and not as exact lifetimes.

\section{The low frequency range $1 \mathrm{nHz} \leq v \leq 30 \mathrm{nHz}$}

The latitude-frequency diagrams of the net and the total magnetic flux are displayed in Figs. 3a and 3b, respectively. The frequency range is $1 \mathrm{nHz} \leq v \leq 30 \mathrm{nHz}$ (corresponding to periods $32 \mathrm{yr} \geq v^{-1} \geq 1 \mathrm{yr}$ ). The color table of each diagram is normalized to the maximum power in the range $10 \mathrm{nHz} \leq v \leq 30 \mathrm{nHz}$, which accentuates the faint features above $10 \mathrm{nHz}$ (the actual power maxima are 23.3 for Fig. $3 \mathrm{a}$ and 117.4 for Fig. 3b). Dotted contour lines indicate the $99.99 \%$ significance level. They correspond to $P_{p=0.9999}$ as defined by Eq. (2) and shown in Figs. 2a and 2b. Regions of special interest are labeled A-D for the net magnetic flux and $\mathrm{A}^{\prime}-\mathrm{D}^{\prime}$ for the total magnetic flux. The corresponding frequencies, periods, latitudes, and time intervals are listed in Table 1, together with the maximum power $P_{\max }$ reached within each region $\left(P_{\max } \geq P_{p}\right)$. We first discuss the results for the total magnetic flux in Sect. 3.1, since they are directly comparable with the results of Paper I. Special emphasis is given to the $1.3 \mathrm{yr}$ periodicity in Sect. 3.1.1. The results for the net magnetic flux are then briefly discussed in Sect. 3.2.

\subsection{The total magnetic flux}

The total magnetic flux in Fig. 3b shows maximum power at $v=3.3 \pm 0.6 \mathrm{nHz}(9.6 \pm 1.7 \mathrm{yr})$, followed by the second harmonic around $v=6.6 \pm 0.6 \mathrm{nHz}(4.8 \pm 0.4 \mathrm{yr})$. The frequency resolution can be artificially increased by zero padding the time series. Power then peaks around $v=3.1 \mathrm{nHz}(10.2 \mathrm{yr})$ and $v=6.2 \mathrm{nHz}(5.1 \mathrm{yr})$. The former corresponds to the $11 \mathrm{yr}$ sunspot cycle, the latter is likely due to the deviation of the $11 \mathrm{yr}$ cycle from a purely sinusoidal shape. Mursula et al. (1997) have shown that a periodicity around 5.5 years may arise from the asymmetry of the cycle shape (i.e. the rise time of a typical solar cycle is shorter than the decline time). 
Table 1. Frequencies $v$, periods $v^{-1}$, maximum power $P_{\max }$, significance levels $p$, and latitudes $\vartheta$ (where power exceeds the given significance level) for the labeled regions in Figs. $3 \mathrm{a}$ and $3 \mathrm{~b}$, respectively. The error $\Delta v=0.6 \mathrm{nHz}$ is half the frequency resolution. The time intervals (last column) were determined using the $99.9 \%$ significance contours of Morlet wavelets with nondimensional frequency 6 or 9 (cf. Sect. 2.2). A time interval in an otherwise empty row belongs to the periodicity above. Rows in brackets indicate periodicities that are evident in the wavelet power spectra (with $99.9 \%$ significance) but not in Figs. 3a and 3b. The frequencies for $\mathrm{A}^{\prime}$ and $\mathrm{B}_{0}^{\prime}$ were computed with a five times higher frequency resolution. The original values calculated with the standard frequency resolution are $8.9 \mathrm{nHz}\left(\mathrm{A}^{\prime}\right)$ and $10.0 \mathrm{nHz}\left(\mathrm{B}_{0}^{\prime}\right)$.

\begin{tabular}{|c|c|c|c|c|c|c|}
\hline & $v(\mathrm{nHz})$ & $v^{-1}(\mathrm{yr})$ & $P_{\max }$ & $p(\%)$ & $\vartheta$ & Time \\
\hline $\bar{A}$ & 7.8 & 4.1 & 1.1 & 99.99 & $21^{\circ} \mathrm{N}-24^{\circ} \mathrm{N}$ & $85.3-02.5$ \\
\hline \multirow{4}{*}{$\mathrm{B}_{1}$} & 12.2 & 2.6 & 1.0 & 99.99 & $26^{\circ} \mathrm{S}-28^{\circ} \mathrm{S}$ & $79.0-82.0$ \\
\hline & & & & & & $87.6-00.9$ \\
\hline & 13.3 & 2.4 & 1.2 & 99.99 & $17^{\circ} \mathrm{S}-18^{\circ} \mathrm{S}$ & $86.0-93.6$ \\
\hline & & $(2.0$ & & 99.90 & $17^{\circ} \mathrm{S}-18^{\circ} \mathrm{S}$ & $99.4-03.6)$ \\
\hline \multirow[t]{2}{*}{$\mathrm{B}_{2}$} & 17.7 & 1.79 & 1.4 & 99.99 & $\sim 18^{\circ} \mathrm{S}$ & $79.5-84.4$ \\
\hline & & & & & & $85.9-92.0$ \\
\hline $\mathrm{B}_{3}$ & 21.1 & 1.50 & 0.6 & 99.00 & $\sim 18^{\circ} \mathrm{S}$ & 88.6-91.6 \\
\hline \multirow[t]{3}{*}{$\mathrm{C}$} & 21.1 & 1.50 & 0.3 & 99.99 & $36^{\circ} \mathrm{S}-47^{\circ} \mathrm{S}$ & $79.0-84.0$ \\
\hline & & & & & & $87.3-92.1$ \\
\hline & & & & & & $98.3-01.6$ \\
\hline \multirow[t]{2}{*}{$\mathrm{D}$} & 23.3 & 1.36 & 0.2 & 99.99 & $39^{\circ} \mathrm{N}-45^{\circ} \mathrm{N}$ & $76.6-81.5$ \\
\hline & & & & & & $89.0-92.5$ \\
\hline$\overline{\mathrm{A}^{\prime}}$ & 9.1 & 3.5 & 4.7 & 99.99 & $16^{\circ} \mathrm{N}-25^{\circ} \mathrm{N}$ & $76.0-91.5$ \\
\hline $\mathrm{B}_{0}^{\prime}$ & 9.3 & 3.4 & 3.9 & 99.99 & $18^{\circ} \mathrm{S}-25^{\circ} \mathrm{S}$ & $86.7-03.5$ \\
\hline \multirow[t]{2}{*}{$\mathrm{B}_{1}^{\prime}$} & 12.2 & 2.6 & 5.0 & 99.99 & $14^{\circ} \mathrm{S}-17^{\circ} \mathrm{S}$ & $86.5-95.0$ \\
\hline & & & & & & $97.7-03.5$ \\
\hline \multirow[t]{2}{*}{$\mathrm{B}_{2}^{\prime}$} & 17.7 & 1.79 & 2.7 & 99.99 & $21^{\circ} \mathrm{S}-25^{\circ} \mathrm{S}$ & $96.5-02.8$ \\
\hline & 18.8 & 1.69 & 3.3 & 99.99 & $\sim 16^{\circ} \mathrm{S}$ & $96.8-03.1$ \\
\hline \multirow[t]{2}{*}{$\mathrm{B}_{3}^{\prime}$} & 22.2 & 1.43 & 2.8 & 99.90 & $12^{\circ} \mathrm{S}-16^{\circ} \mathrm{S}$ & $79.3-84.7$ \\
\hline & & $(1.57$ & & 99.90 & $9^{\circ} \mathrm{N}-16^{\circ} \mathrm{N}$ & $78.5-85.1)$ \\
\hline \multirow[t]{3}{*}{$\mathrm{C}^{\prime}$} & 24.4 & 1.30 & 0.1 & 99.99 & $42^{\circ} \mathrm{S}-54^{\circ} \mathrm{S}$ & 86.6-94.4 \\
\hline & & & & & & $98.2-02.4$ \\
\hline & & $(1.32$ & & 99.90 & $30^{\circ} \mathrm{S}-42^{\circ} \mathrm{S}$ & 88.4-93.8) \\
\hline \multirow[t]{5}{*}{$\mathrm{D}^{\prime}$} & 24.4 & 1.30 & 0.9 & 99.99 & $30^{\circ} \mathrm{N}-36^{\circ} \mathrm{N}$ & $77.0-80.2$ \\
\hline & & & & & & $87.0-93.0$ \\
\hline & & & & & & $98.5-01.0$ \\
\hline & & (1.32 & & 99.90 & $36^{\circ} \mathrm{N}-52^{\circ} \mathrm{N}$ & $75.8-80.9)$ \\
\hline & & $(1.32$ & & 99.90 & $36^{\circ} \mathrm{N}-52^{\circ} \mathrm{N}$ & 89.1-93.4) \\
\hline
\end{tabular}

Significant power is also seen at higher frequencies, for instance in the regions $\mathrm{A}^{\prime}$ in the north and $\mathrm{B}_{0}^{\prime}$ in the south. The corresponding frequencies (and periods) are $v=8.9 \pm 0.6 \mathrm{nHz}$ $(3.6 \pm 0.2 \mathrm{yr})$ and $v=10.0 \pm 0.6 \mathrm{nHz}(3.2 \pm 0.2 \mathrm{yr})$, respectively. Increased frequency resolution yields $v=9.1 \mathrm{nHz}$ (3.5 yr) for $\mathrm{A}^{\prime}$ and $v=9.3 \mathrm{nHz}\left(3.4 \mathrm{yr}\right.$ ) for $\mathrm{B}_{0}^{\prime}$. We cannot exclude the possibility that also these frequencies may be harmonics of the sunspot cycle. On the other hand, wavelet analysis showed that the oscillation associated with $\mathrm{A}^{\prime}$ occurred in the total flux approximately during 1976-1992, while that associated with $\mathrm{B}_{0}^{\prime}$ occurred during 1987-2003. In Paper I we have shown that a $3.6 \pm 0.3 \mathrm{yr}$ periodicity was present in the $\mathrm{N}-\mathrm{S}$ asymmetry of the total flux from 1984 to 1995, so we now have reason to believe that this periodicity is due to $\mathrm{A}^{\prime}$ and $\mathrm{B}_{0}^{\prime}$. Moreover, Berdyugina \& Usoskin (2003) have analyzed active longitudes in sunspot activity over the last 120 years and found a persistent $3.8 \mathrm{yr}$ periodicity in the north and a $3.65 \mathrm{yr}$ periodicity in the south. A $3.7 \mathrm{yr}$ periodicity has been reported by Joshi \& Joshi (2004) in the N-S asymmetry of the soft X-ray flare index during cycles 21-23, periodicities around 3.1-3.2 yr were detected by Vizoso \& Ballester (1989) in the N-S asymmetry of flare indices, and periodicities of 3.5-3.9 yr were found by Rao (1973) in spot groups indices. These studies support the view that periodicities in the range of 3-4 yr may be of solar origin.

The regions $\mathrm{B}_{1}^{\prime}, \mathrm{B}_{2}^{\prime}$ and $\mathrm{B}_{3}^{\prime}$ correspond to oscillations that are often referred to as "quasi-biennial" (Benevolenskaya 1998; Bazilevskaya et al. 2000; Obridko \& Shelting 2001; Dzhalilov et al. 2002). The dominant peak in $\mathrm{B}_{1}^{\prime}$ has the frequency $v=12.2 \pm 0.6 \mathrm{nHz}(2.6 \pm 0.1 \mathrm{yr})$ and the associated oscillation occurred approximately during 1987-1995 and 1998-2003. The region $\mathrm{B}_{2}^{\prime}$ contains significant power at the frequencies $v=17.7 \pm 0.6 \mathrm{nHz}(1.79 \pm 0.06 \mathrm{yr})$ and $v=18.8 \pm$ $0.6 \mathrm{nHz}(1.68 \pm 0.05 \mathrm{yr})$. Both oscillations were present during 1997-2003. This is in good agreement with the $1.79 \mathrm{yr}$ and $1.65 \mathrm{yr}$ periodicities reported in Paper I for the same time interval. Finally, the oscillation associated with the region $\mathrm{B}_{3}^{\prime}$ at $v=22.3 \pm 0.6 \mathrm{nHz}(1.43 \pm 0.04 \mathrm{yr})$ occurred in the latitude zone $12^{\circ} \mathrm{S}-16^{\circ} \mathrm{S}$ from 1979 to 1985 . Although not clearly visible in Fig. 3b, additional wavelet analysis showed significant power at a period of $1.57 \mathrm{yr}$ in the latitude band $9^{\circ} \mathrm{N}-16^{\circ} \mathrm{N}$ over the same time interval (cf. Table 1). These two periodicities are therefore likely to be the source of the $1.50 \mathrm{yr}$ period in the N-S asymmetry of the total flux described in Paper I. The circumstance that two oscillations with similar periods around $1.5 \mathrm{yr}$ (but with different phases) occurred in both hemispheres during cycle 21 was also noted by Ichimoto et al. (1985) in their analysis of $\mathrm{H} \alpha$ flares.

\subsubsection{The 1.3-year periodicity}

Interestingly, regions $\mathrm{C}^{\prime}$ and $\mathrm{D}^{\prime}$ in Fig. 3b show significant power at $v=24.4 \pm 0.6 \mathrm{nHz}(1.30 \pm 0.03 \mathrm{yr})$ in both hemispheres. The latitude bands where the power exceeds the $99.99 \%$ significance level are $42^{\circ}-54^{\circ} \mathrm{S}$ and $30^{\circ}-36^{\circ} \mathrm{N}$. Figure 4 shows the total magnetic flux averaged over these two latitude bands as a function of time and the corresponding wavelet power spectra. It can be clearly seen that there is significant power around the $1.3 \mathrm{yr}$ periodicity during the maxima of cycles 21-23. During cycle 22, it was prominent in both hemispheres from 1987 to 1994 , although the periods were slightly shorter (1.22 yr in the north, $1.26 \mathrm{yr}$ in the south). Additional wavelet analysis showed that the $1.3 \mathrm{yr}$ period was present over the latitude zones $\sim 30^{\circ}-55^{\circ}$ in both hemispheres. We note that the $1.3 \mathrm{yr}$ period cannot be detected if the total magnetic flux is averaged over all latitudes, since the signal is relatively weak.

Howe et al. (2000) have reported a $1.3 \mathrm{yr}$ period in the variation of the rotation rate near the base of the convection zone from 1995 to 1999 , being most pronounced at low and 

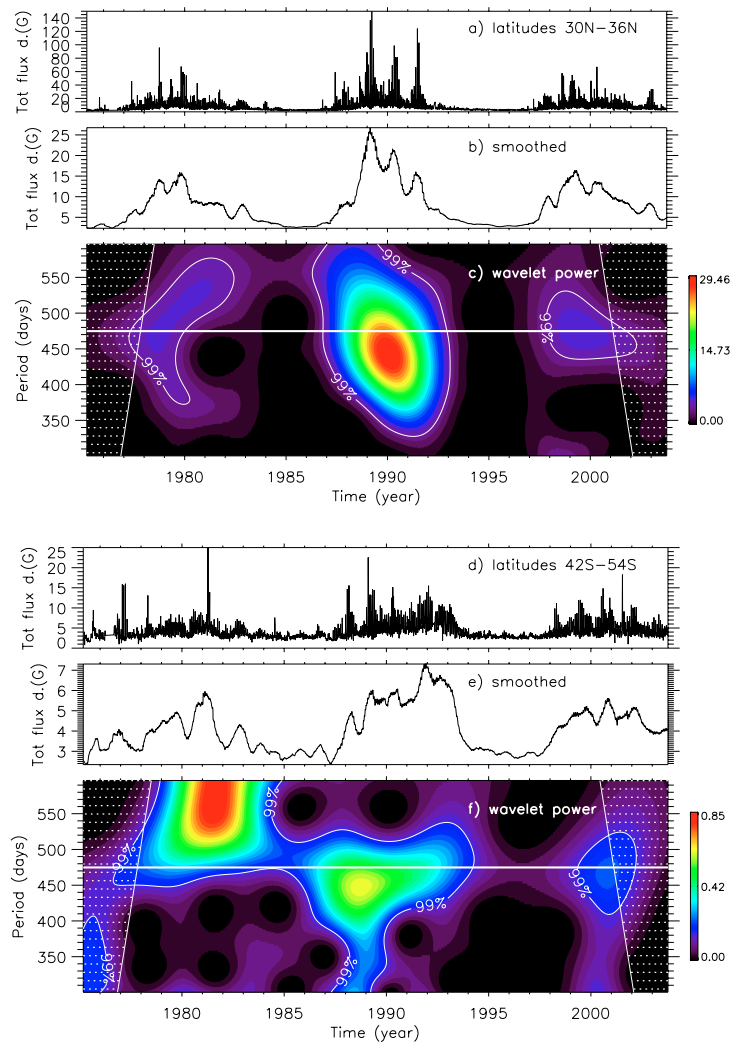

Fig. 4. a) The raw time series of the total magnetic flux density averaged over latitudes $30^{\circ}-36^{\circ} \mathrm{N}$. b) The smoothed time series calculated with an averaging window of 6 months. c) The wavelet power spectrum of the raw time series, calculated with Morlet wavelets and nondimensional frequency 9 (cf. Sect. 2.2). It clearly shows enhanced power at the 99\% significance level (white contour lines) around a periodicity of $1.3 \mathrm{yr}$ (represented by the horizontal white line at 475 days) during all three cycles 21-23. The hatched areas indicate the "cone of influence", where edge effects become important. d)-f) The same for latitudes $42^{\circ}-54^{\circ} \mathrm{S}$. The wavelet power spectrum shows significant power around $1.3 \mathrm{yr}$ for cycles 22 and 23.

intermediate latitudes. Toomre et al. (2003) have shown that the variations near the equator diminished in amplitude when solar cycle 23 attained its maximum.

It is not clear whether the $1.3 \mathrm{yr}$ periodicity in the rotation rate at the base of the convection zone is related to the $1.3 \mathrm{yr}$ periodicity in the photospheric magnetic flux. If there is such a relationship, then the disappearance of the $1.3 \mathrm{yr}$ oscillation in the photosphere after 2002 (as seen in Fig. 4) may possibly be linked to the damping of the $1.3 \mathrm{yr}$ oscillation near the tachocline.

Periodicities at $1.3 \mathrm{yr}$ have previously been reported in the photosphere and the heliosphere by various authors. Krivova \& Solanki (2002) found variations with this period in the sunspot areas and sunspot numbers. These variations were particularly dominant during the interval 1920-1965 and appeared again, although considerably weaker, in the 21 st and subsequent cycles. Lockwood (2001) found the $1.3 \mathrm{yr}$ period in the interplanetary magnetic field (IMF) and geomagnetic activity (see also Mursula et al. 2003). Lockwood (2001) noted that the $1.3 \mathrm{yr}$ period has been present mainly since 1940, with the largest amplitudes near the maxima of even-numbered sunspot cycles.
This is in good agreement with Fig. 4, which shows that the $1.3 \mathrm{yr}$ periodicity was most dominant during cycle 22 . In addition, Richardson et al. (1994) and Gazis et al. (1995) detected a 1.3 yr period in the solar wind speed during 1987-1994, while Szabo et al. (1995) found it in the southward component of the IMF. Besides, the $1.3 \mathrm{yr}$ period has also been seen in geomagnetic (Paularena et al. 1995) and auroral phenomena (Silverman \& Shapiro 1983).

The $1.3 \mathrm{yr}$ period in the unsigned (and signed) magnetic flux occurs at latitudes where large-scale magnetic surges towards the poles (Howard \& Labonte 1981; Wang et al. 1989) seem to emanate. This can be nicely seen in the butterfly diagram of the signed flux in Fig. 1a (the periodicities for the signed flux are slightly larger, around 1.4-1.5 yr). We therefore propose that the $\sim 1.3 \mathrm{yr}$ periodicity in the photospheric magnetic flux, the IMF and geomagnetic activity is linked to these magnetic surges. Wang \& Sheeley (2003) have shown that stochastic processes may provide a viable explanation for quasi-periodicities in the range $\sim 1-3 \mathrm{yr}$. However, since our results indicate that the $1.3 \mathrm{yr}$ periodicity has been a feature of all three sunspot cycles 21-23, it appears unlikely that this period is a random occurrence. It will be interesting so see whether the $1.3 \mathrm{yr}$ period will be prominent again during the next cycle 24 .

\subsection{The net magnetic flux}

For the net magnetic flux in Fig. 3a, most power is obviously concentrated at the frequency $v=1.1 \pm 0.6 \mathrm{nHz}$ (corresponding to a period of $29 \pm 16 \mathrm{yr}$ ). The latitude zones are $\sim 0^{\circ}-20^{\circ}$ and $\sim 30^{\circ}-90^{\circ}$ in both hemispheres. Increasing the frequency resolution yields $v=1.5 \pm 0.1 \mathrm{nHz}(21.1 \pm 1.4 \mathrm{yr})$, which clearly represents the Hale magnetic cycle with an average period of $22 \mathrm{yr}$. Increased power is also seen at the harmonic $v=4.4 \pm 0.6 \mathrm{nHz}(7.2 \pm 1.0 \mathrm{yr})$. Zeropadding yields $v \approx 4.7 \mathrm{nHz}(6.7 \mathrm{yr})$. This harmonic may be due to deviations of the $22 \mathrm{yr}$ cycle from a purely sinusoidal shape.

As for the total flux, there is increased power at higher frequencies, mostly in similar regions. Thus, we will discuss these periods only briefly. The dominant peak in region A extends over latitudes $21^{\circ}-24^{\circ} \mathrm{N}$ and has the frequency $v=7.8 \pm$ $0.6 \mathrm{nHz}(4.1 \pm 0.3 \mathrm{yr})$. In contrast to the total magnetic flux, there seems to be no southern analogue to region A in Fig. 3a. The regions $B_{1}-B_{3}$ in Fig. 3a appear at approximately the same frequencies as the regions $B_{1}^{\prime}-B_{3}^{\prime}$ in Fig. $3 b$. Note that region $B_{3}$ is weak compared to $\mathrm{B}_{3}^{\prime}$. Finally, the regions $\mathrm{C}$ and $\mathrm{D}$ appear at slightly different latitudes and lower frequencies than the analogous regions $\mathrm{C}^{\prime}$ and $\mathrm{D}^{\prime}$ (cf. Table 1). The corresponding oscillations occurred during all three cycles 21-23. As already pointed out in the previous section, they appear to be directly linked to the large-scale magnetic surges towards the poles.

\section{The intermediate frequency range $30 \mathrm{nHz} \leq v \leq 120 \mathrm{nHz}$}

Figures $5 \mathrm{a}$ and $5 \mathrm{~b}$ show the frequency-latitude diagrams of the net and the total magnetic flux, respectively, for the intermediate frequency range $30 \mathrm{nHz} \leq v \leq 120 \mathrm{nHz}$ (386 d $\left.\geq v^{-1} \geq 96 \mathrm{~d}\right)$. Dotted contour lines indicate the 

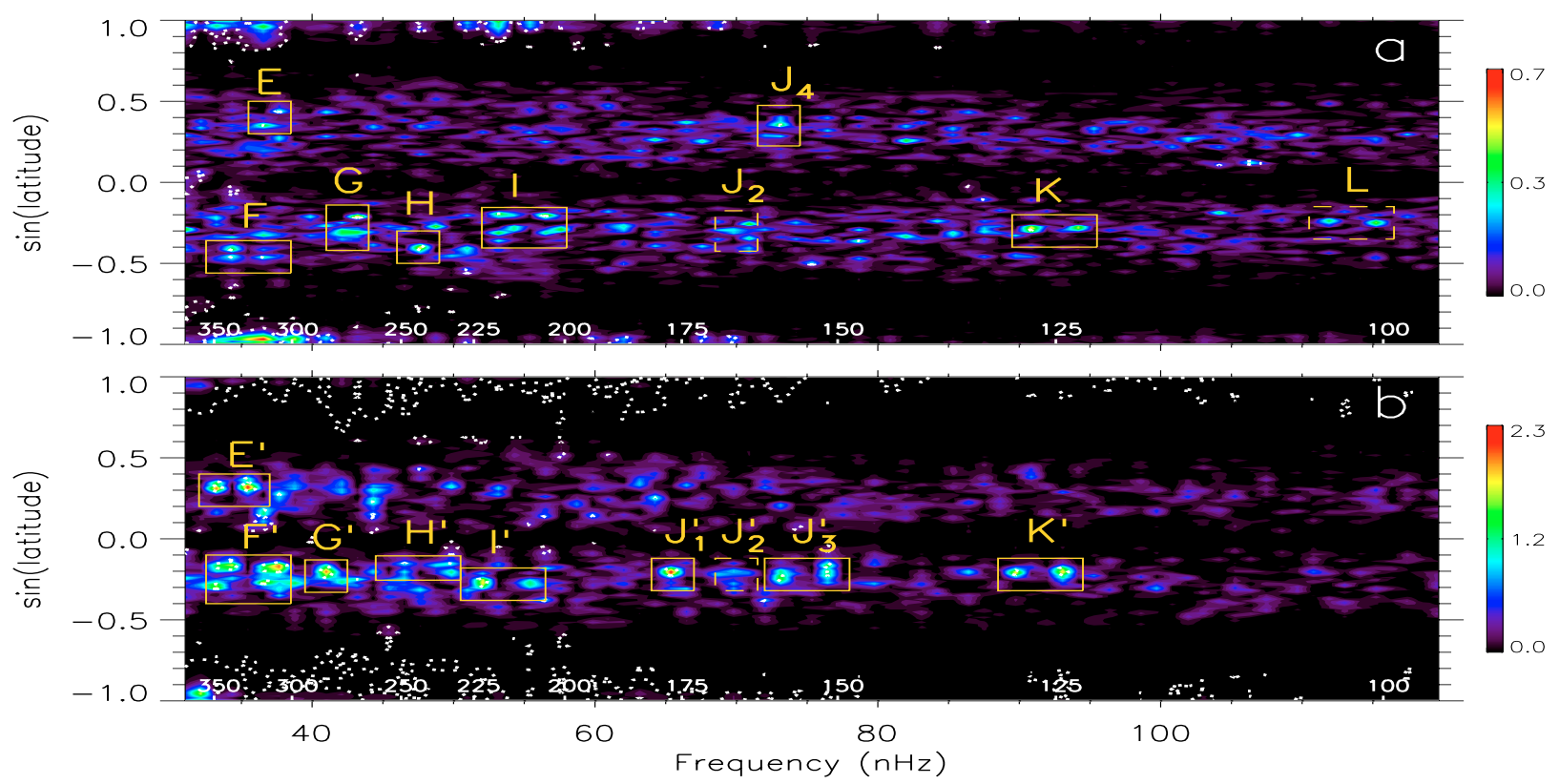

Fig. 5. a) FFT power density (in arbitrary but constant units) vs. sine latitude and frequency for the net (signed) magnetic flux. The frequency range is 30-120 nHz (corresponding to periods 386-96 d, indicated in white on the horizontal axis). Dotted contour lines represent the 99.0\% significance level. The labeled regions are listed in Table 2. b) The same for the total (unsigned) magnetic flux.

99.0\% significance level and correspond to $P_{p=0.99}$ (as defined by Eq. (2) and shown in Figs. 2a and 2b). Regions of special interest are labeled $\mathrm{E}-\mathrm{L}$ for the net magnetic flux and $\mathrm{E}^{\prime}-\mathrm{K}^{\prime}$ for the total magnetic flux. The corresponding frequencies, periods, maximum power, latitudes, and time intervals are listed in Table 2. Generally, the results for both the total magnetic flux and the net magnetic flux are in qualitative agreement although some discrepancies exist. In the following, we will mainly concentrate on the results for the total magnetic flux and only briefly discuss those for the net magnetic flux.

Interestingly, the total magnetic flux in Fig. 5b exhibits a whole series of peaks that are confined to the latitude zone $\sim 10^{\circ}-20^{\circ}$ in the southern hemisphere. Several of them are double peaks separated by $\Delta v=v_{2}-v_{1} \approx 3.4 \mathrm{nHz}(\Delta v \approx 9.3 \mathrm{yr})$, where $v_{1}$ and $v_{2}$ are the frequencies of the single peaks. These double peaks are the consequence of amplitude modulation by the underlying solar cycle, which implies that the "true" frequency of the corresponding oscillation is $\left(v_{1}+v_{2}\right) / 2$. In the following, we will use a hyphen to indicate the frequency range of peaks that cluster in a narrow frequency interval, while a " \pm " indicates the error of single (isolated) peaks.

The region $\mathrm{F}^{\prime}$ contains two double peaks in the range $33.2-36.6 \mathrm{nHz}(349-316 \mathrm{~d})$ and $34.3-37.7 \mathrm{nHz}(337-307 \mathrm{~d})$. Further peaks occur in regions $\mathrm{G}^{\prime}$ at $41.0 \pm 0.6 \mathrm{nHz}(282 \pm$ $4 \mathrm{~d}), \mathrm{H}^{\prime}$ at $46.5-49.9 \mathrm{nHz}(249-232 \mathrm{~d}), \mathrm{I}^{\prime}$ at $52.1-55.4 \mathrm{nHz}$ $(222-209, \mathrm{~d}), \mathrm{J}^{\prime}{ }_{1}$ at $65.4 \pm 0.6 \mathrm{nHz}(177 \pm 2 \mathrm{~d}), \mathbf{J}^{\prime}{ }_{3}$ at $73.1-76.5 \mathrm{nHz}(158-151 \mathrm{~d})$ and $\mathrm{K}^{\prime}$ at $89.7-93.1 \mathrm{nHz}$ (129-124 d). Two additional peaks are seen in the northern region $\mathrm{E}^{\prime}$ at $33.2-35.5 \mathrm{nHz}$ (349-326 d). The net magnetic flux in Fig. 5a additionally shows a significant peak in the northern region $\mathrm{J}_{4}$ at $73.1 \pm 0.6 \mathrm{nHz}(158 \pm 1 \mathrm{~d})$ and a double peak in the southern region $\mathrm{L}$ at $111.9-115.2 \mathrm{nHz}(103-100 \mathrm{~d})$.

\subsection{The Rieger period}

The best known of these periodicities is the Rieger period around 155 days, which was first observed in the occurrence of high-energy flares from 1980 to 1984 (Rieger et al. 1984; Kiplinger et al. 1984; Bogart \& Bai 1985) and has since been found in various indicators of solar activity (see, for example, Bai 2003b, and references therein). Analyses of sunspot areas and sunspot numbers of recent cycles have shown that the Rieger period was present during cycles 16-21 (Lean \& Brueckner 1989; Lean 1990; Carbonell \& Ballester 1992; Oliver et al. 1998; Krivova \& Solanki 2002), but not during cycles 12-15 (Carbonell \& Ballester 1992). Oliver et al. (1998) and Ballester et al. (2002) have argued that the Rieger period disappeared after cycle 21 , which is supported by a recent analysis of periodicities in solar flare occurrence during cycles $19-23$ by Bai (2003b). However, Krivova \& Solanki (2002) have found evidence that it persisted in sunspots during cycle 22, and Ballester et al. (2004) have detected it again during cycle 23 .

The wavelet power spectrum of the total magnetic flux averaged over latitudes $10^{\circ}-20^{\circ}$ in each hemisphere is displayed in Fig. 6. It shows that the Rieger period occurred in the south during cycles 21 and 22 (Fig. 6c), but only for a very limited time interval. No significant power at this periodicity is seen in the north (Fig. 6b). If the unsigned flux is averaged over latitudes $20^{\circ} \mathrm{S}-20^{\circ} \mathrm{N}$, the wavelet power spectrum shows a significant peak at the Rieger period during cycle 21 and a less significant peak during cycle 22 (Fig. 6a). Thus, we can support the conclusions by Krivova \& Solanki (2002) that the Rieger period was present during cycle 22 , although predominantly in the south. 
Table 2. Frequencies $v$, periods $v^{-1}$, maximum power $P_{\max }$, significance levels $p$, and latitudes $\vartheta$ (where the power exceeds the given significance level) for the labeled regions in Figs. 5a and 5b. Asterisks indicate that the 99.9\% level was also reached. The error $\Delta v=0.6 \mathrm{nHz}$ is half the frequency resolution. The time intervals (last column) were determined using the $99.0 \%$ significance contours of Morlet wavelets with nondimensional frequency 12 (cf. Sect. 2.2). The time interval for the peak at $33.2 \mathrm{nHz}$ in region $\mathrm{F}^{\prime}$ could not be determined unambiguously.

\begin{tabular}{|c|c|c|c|c|c|c|c|c|c|c|c|c|c|}
\hline & $v(\mathrm{nHz})$ & $v^{-1}(\mathrm{~d})$ & $P_{\max }$ & $p(\%)$ & $\vartheta$ & Time & & $v(\mathrm{nHz})$ & $v^{-1}(\mathrm{~d})$ & $P_{\max }$ & $p(\%)$ & $\vartheta$ & Time \\
\hline \multirow[t]{2}{*}{$\mathrm{E}$} & 36.6 & 316 & 0.44 & 99.0 & $20^{\circ} \mathrm{N}$ & $78-82$ & \multirow[t]{2}{*}{$\mathrm{E}^{\prime}$} & 33.2 & 349 & 2.1 & $99.0^{*}$ & $17^{\circ} \mathrm{N}-20^{\circ} \mathrm{N}$ & $88-92$ \\
\hline & 37.7 & 307 & 0.35 & 99.0 & $26^{\circ} \mathrm{N}$ & 80-81/99-02 & & 35.5 & 326 & 2.3 & $99.0 *$ & $18^{\circ} \mathrm{N}-22^{\circ} \mathrm{N}$ & $98-02$ \\
\hline \multirow[t]{4}{*}{$\mathrm{F}$} & 34.3 & 337 & 0.34 & 99.0 & $27^{\circ} \mathrm{S}$ & 88-92/98-01 & \multirow[t]{4}{*}{$\mathrm{F}^{\prime}$} & 33.2 & 349 & 1.7 & 99.0 & $16^{\circ} \mathrm{S}-18^{\circ} \mathrm{S}$ & - \\
\hline & 34.3 & 337 & 0.43 & 99.0 & $25^{\circ} \mathrm{S}$ & $88-92$ & & 34.3 & 337 & 1.8 & $99.0 *$ & $9^{\circ} \mathrm{S}-11^{\circ} \mathrm{S}$ & $81-85 / 91-93$ \\
\hline & 36.6 & 316 & 0.36 & 99.0 & $27^{\circ} \mathrm{S}$ & 88-92/98-01 & & 36.6 & 316 & 1.8 & 99.0 & $15^{\circ} \mathrm{S}-16^{\circ} \mathrm{S}$ & $79-82$ \\
\hline & & & & & & & & 37.7 & 307 & 2.1 & $99.0 *$ & $9^{\circ} \mathrm{S}-12^{\circ} \mathrm{S}$ & $80-85$ \\
\hline \multirow[t]{2}{*}{ G } & 42.1 & 275 & 0.41 & 95.0 & $17^{\circ} \mathrm{S}-19^{\circ} \mathrm{S}$ & 89-91/02-03 & \multirow[t]{2}{*}{$\mathrm{G}^{\prime}$} & \multirow[t]{2}{*}{41.0} & \multirow[t]{2}{*}{282} & \multirow[t]{2}{*}{2.1} & \multirow[t]{2}{*}{$99.0 *$} & \multirow[t]{2}{*}{$10^{\circ} \mathrm{S}-14^{\circ} \mathrm{S}$} & \multirow{2}{*}{$90-94$} \\
\hline & 43.2 & 268 & 0.62 & 99.0 & $12^{\circ} \mathrm{S}-13^{\circ} \mathrm{S}$ & $81-82 / 00-01$ & & & & & & & \\
\hline \multirow[t]{2}{*}{$\mathrm{H}$} & 47.6 & 243 & 0.56 & 99.0 & $23^{\circ} \mathrm{S}-25^{\circ} \mathrm{S}$ & $90-93$ & \multirow[t]{2}{*}{$\mathrm{H}^{\prime}$} & 46.5 & 249 & 1.0 & 99.0 & $\sim 9^{\circ} \mathrm{S}$ & $91-92 / 01-03$ \\
\hline & & & & & & & & 49.9 & 232 & 1.0 & 99.0 & $\sim 9^{\circ} \mathrm{S}$ & $81-84$ \\
\hline \multirow[t]{5}{*}{ I } & 53.2 & 218 & 0.44 & 99.0 & $11^{\circ} \mathrm{S}$ & $90-93$ & \multirow[t]{4}{*}{$\mathrm{I}^{\prime}$} & 52.1 & 222 & 1.9 & 99.0 & $14^{\circ} \mathrm{S}-17^{\circ} \mathrm{S}$ & 80-82/89-90 \\
\hline & 53.2 & 218 & 0.42 & 95.0 & $16^{\circ} \mathrm{S}-18^{\circ} \mathrm{S}$ & $81-82 / 88-90$ & & 55.4 & 209 & 1.3 & 95.0 & $14^{\circ} \mathrm{S}-17^{\circ} \mathrm{S}$ & $01-02$ \\
\hline & 56.5 & 205 & 0.55 & 99.0 & $11^{\circ} \mathrm{S}$ & $82-84$ & & & & & & & \\
\hline & 56.5 & 205 & 0.35 & 95.0 & $16^{\circ} \mathrm{S}-18^{\circ} \mathrm{S}$ & $81-82 / 88-90$ & & & & & & & \\
\hline & & & & & & & $\mathrm{J}_{1}^{\prime}$ & 65.4 & 177 & 2.3 & $99.0 *$ & $11^{\circ} \mathrm{S}-13^{\circ} \mathrm{S}$ & $90-93$ \\
\hline \multirow[t]{5}{*}{$\mathrm{J}_{2}$} & 70.9 & 163 & 0.47 & 95.0 & $14^{\circ} \mathrm{S}-15^{\circ} \mathrm{S}$ & 81-83/89-90 & \multirow[t]{2}{*}{$\mathrm{J}_{2}^{\prime}$} & \multirow[t]{2}{*}{69.8} & \multirow[t]{2}{*}{166} & \multirow[t]{2}{*}{0.9} & \multirow[t]{2}{*}{95.0} & \multirow[t]{2}{*}{$11^{\circ} \mathrm{S}-13^{\circ} \mathrm{S}$} & \multirow[t]{2}{*}{$00-01$} \\
\hline & 70.9 & 163 & 0.35 & 95.0 & $19^{\circ} \mathrm{S}-20^{\circ} \mathrm{S}$ & $90-91 / 01-03$ & & & & & & & \\
\hline & & & & & & & $\mathrm{J}_{3}^{\prime}$ & 73.1 & 158 & 1.6 & 99.0 & $13^{\circ} \mathrm{S}-15^{\circ} \mathrm{S}$ & $80-82 / 90-93$ \\
\hline & & & & & & & & 76.5 & 151 & 1.4 & 99.0 & $9^{\circ} \mathrm{S}-12^{\circ} \mathrm{S}$ & $81-82 / 90-92$ \\
\hline & & & & & & & & 76.5 & 151 & 1.4 & 99.0 & $3^{\circ} \mathrm{S}-5^{\circ} \mathrm{S}$ & $90-93$ \\
\hline $\mathrm{J}_{4}$ & 73.1 & 158 & 0.42 & 99.0 & $21^{\circ} \mathrm{N}$ & 00-02 & & & & & & & \\
\hline \multirow[t]{2}{*}{$\mathrm{K}$} & 90.9 & 127 & 0.57 & 99.0 & $16^{\circ} \mathrm{S}-17^{\circ} \mathrm{S}$ & 89-92/02-03 & \multirow[t]{4}{*}{$\mathrm{K}^{\prime}$} & 89.7 & 129 & 1.9 & 99.0 & $11^{\circ} \mathrm{S}-13^{\circ} \mathrm{S}$ & $80-81 / 90-93$ \\
\hline & 94.2 & 123 & 0.49 & 95.0 & $16^{\circ} \mathrm{S}-17^{\circ} \mathrm{S}$ & 89-92/02-03 & & 93.1 & 124 & 1.9 & 99.0 & $11^{\circ} \mathrm{S}-13^{\circ} \mathrm{S}$ & $80-81 / 90-93$ \\
\hline \multirow[t]{2}{*}{$\mathrm{L}$} & 111.9 & 103 & 0.40 & 95.0 & $14^{\circ} \mathrm{S}$ & $81-83$ & & & & & & & \\
\hline & 115.2 & 100 & 0.47 & 95.0 & $14^{\circ} \mathrm{S}-15^{\circ} \mathrm{S}$ & $81-83$ & & & & & & & \\
\hline
\end{tabular}

As far as cycle 23 is concerned, we find a less significant peak $\left(\mathbf{J}_{2}^{\prime}\right)$ at the periodicity of $166 \pm 1$ days, which was present in the south during 2000-2001. This is supported by the results for the net flux. The peaks in region $\mathrm{J}_{2}$ in Fig. 5a correspond to a period of $163 \pm 1$ days, which occurred during all three cycles 21-23 (cf. Table 2). Peak $\mathrm{J}_{4}$ at the period of $158 \pm 1$ days indicates that the Rieger period was present also in the north during 2000-2002. This peak is however seen only in the net flux but not in the total flux. Since the data for cycle 23 are still incomplete, these results are preliminary and should be used with caution. Zięba et al. (2001) found the Rieger period in the ascending phase of cycle 23 in solar radio flux data and sunspot numbers, but not in the mean magnetic field.

\subsection{Rieger-type periodicities}

Midterm periodicities in the range of 50-350 days other than the Rieger period have been reported before in various studies of solar activity indices. Let us here briefly summarize some of the relevant results. Periods near $320 \mathrm{~d}$ were found in the Zurich sunspot number from 1749 to 1979 (Wolff 1983), in solar diameter measurements from 1975 to 1983 (Delache et al. 1985), in the sunspot blocking function, $10.7 \mathrm{~cm}$ radio flux, sunspot number, and plage index (but only during cycle 21 , cf.
Lean \& Brueckner 1989), in sunspot areas and sunspot numbers (Oliver et al. 1992), and also in the north-south asymmetry of sunspot areas during several solar activity minima (Paper I). Pap et al. (1990) reported, for instance, periodicities near 315, 227, 157, 113 and $50 \mathrm{~d}$ for both the Nimbus-7/ERB (1978-1988) and SMM/ACRIM (1980-1988) total irradiance data. Periods near 330, 287, 237, 155 and $51 \mathrm{~d}$ were found in the $10.7 \mathrm{~cm}$ flux (1947-1989), and periods near 350, 280, 250 and $156 \mathrm{~d}$ in the sunspot blocking function (1874-1982). In solar flare occurrence, Bai (2003b) reported, beside the Rieger period, periodicities of $51 \mathrm{~d}$ (cycle 19), $78 \mathrm{~d}$ (cycle 20), $84 \mathrm{~d}$ (cycle 20), and 129 d (cycles 20 and 23). Finally, Lou et al. (2003) detected for the time interval 1999-2003 periodicities of, for example, $358 \pm 38,272 \pm 26,196 \pm 13,100 \pm 3$ and $66 \pm$ $1 \mathrm{~d}$ for daily counts of CME events (recorded with the Large Angle Spectrometric Coronograph LASCO on board SOHO) and periods of $260 \pm 24,157 \pm 11,122 \pm 5$ and $98 \pm 3 d$ for solar X-ray flares.

A common feature of most of these reported midterm periodicities is that they are close to integer multiples of a period around 25-26 d, which itself is near the sidereal solar rotation period at the equator. We use the expression "Rieger-type" for such periodicities (Lou 2000). The hypothesis that a fundamental period could cause the excitation of subharmonic 


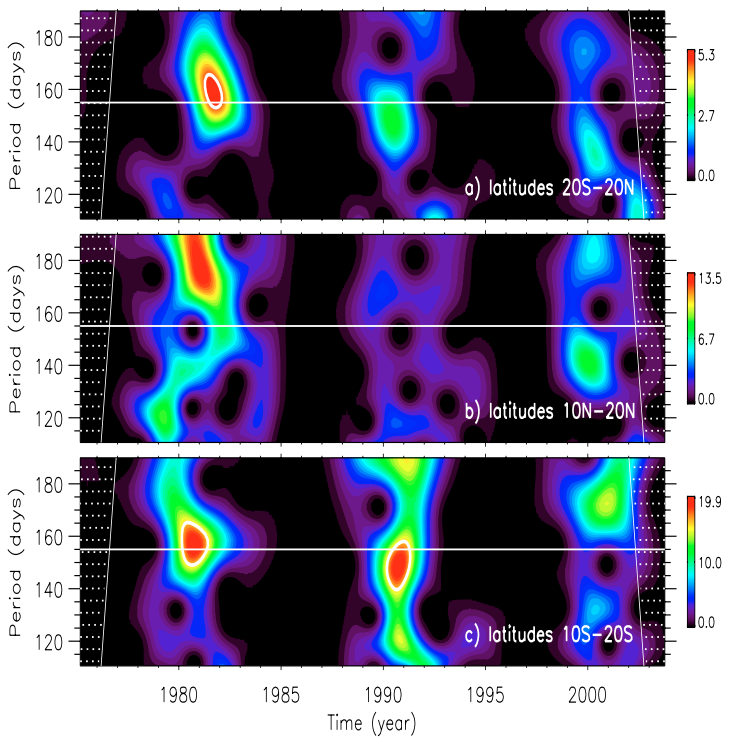

Fig. 6. a) Wavelet power spectrum of the total magnetic flux averaged over latitudes $20^{\circ} \mathrm{S}-20^{\circ} \mathrm{N}$. Morlet wavelets with nondimensional frequency 12 were applied. The Rieger period around $155 \mathrm{~d}$ is indicated by the horizontal white line. Significant power at the Rieger period is seen around 1981-1982. The white contour line corresponds to the $99.0 \%$ significance level. b) The same for latitudes $10^{\circ} \mathrm{N}-20^{\circ} \mathrm{N}$. c) The same for latitudes $10^{\circ} \mathrm{S}-20^{\circ} \mathrm{S}$. Significant power at the Rieger period is seen for cycles 21 and 22 .

oscillations (i.e. oscillations with periods close to integer multiples of the fundamental period) was first formulated by Bai \& Sturrock (1991). The authors showed that periodicities of 51, 78, 104, 129 and $154 \mathrm{~d}$ intermittently occurred in flare and sunspot records during 1955-1985 and therefore inferred that these periods were subharmonics of a fundamental period around 25.8 d. Sturrock \& Bai (1992) and Bai \& Sturrock (1993) proposed an obliquely rotating structure or wave pattern as a possible mechanism for this fundamental period and modified its value to 25.5 d. However, Goode \& Thompson (1992), Gough \& Kosovichev (1992), and Gough et al. (1995) showed that such a model would be seriously constrained by helioseismological data. Direct evidence for a fundamental $25.5 \mathrm{~d}$ period was reported by Ozgüç \& Ataç (1996) for the northsouth asymmetry of flares during cycle 22 . Recently, it has been pointed out (Sturrock et al. 1999; Lou 2000; Sturrock 2004) that the Rieger and Rieger-type periodicities may be due to Rossby-type waves. We will return to this point in Sect. 6.

\section{The high frequency range $390 \mathrm{nHz} \leq v \leq 470 \mathrm{nHz}$}

The latitude-frequency diagrams of the net and total magnetic flux for the high frequency range $390 \mathrm{nHz} \leq v \leq 470 \mathrm{nHz}$ ( $29.7 \mathrm{~d} \geq v^{-1} \geq 24.6 \mathrm{~d}$ ) are displayed in Figs. $7 \mathrm{a}$ and $7 \mathrm{~b}$, respectively. The two diagrams show the spectral power density calculated for the entire time series (cycles 21, 22, and 23). Dotted contour lines indicate the $99.99 \%$ significance level $\left(P_{p=0.9999}\right.$, cf. Eq. (2) and Figs. 2a, 2b). Frequencies, periods, latitudes and time intervals of labeled peaks (N-U for the net flux, $\mathbf{M}^{\prime}-\mathrm{U}_{2}^{\prime}$ for the total flux) are listed in Table 3. The
Table 3. Frequencies $v$, periods $v^{-1}$, maximum Power $P_{\max }$, significance levels $p$, latitudes $\vartheta$ (where the power exceeds the given significance level) and cycles (when the corresponding oscillations occurred) for the labeled regions in Figs. 7a and 7b. The error $\Delta v=0.6 \mathrm{nHz}$ is half the frequency resolution.

\begin{tabular}{|c|c|c|c|c|c|c|}
\hline & $v(\mathrm{nHz})$ & $v^{-1}(\mathrm{~d})$ & $P_{\max }$ & $p(\%)$ & $\vartheta$ & Cycle \\
\hline $\mathrm{N}$ & 400.0 & 28.9 & 1.1 & 99.99 & $27^{\circ} \mathrm{S}-58^{\circ} \mathrm{S}$ & 23 \\
\hline $\mathrm{O}$ & 401.1 & 28.9 & 0.4 & 99.99 & $34^{\circ} \mathrm{S}-54^{\circ} \mathrm{S}$ & $21,22,23$ \\
\hline $\mathrm{P}$ & 406.6 & 28.5 & 0.6 & 99.99 & $28^{\circ} \mathrm{S}-54^{\circ} \mathrm{S}$ & 23 \\
\hline Q & 406.6 & 28.5 & 1.2 & 99.99 & $26^{\circ} \mathrm{N}-57^{\circ} \mathrm{N}$ & 22 \\
\hline \multirow[t]{2}{*}{$\mathrm{R}$} & 411.0 & 28.2 & 1.1 & 99.99 & $19^{\circ} \mathrm{S}-57^{\circ} \mathrm{S}$ & 21,22 \\
\hline & 414.4 & 27.9 & 1.0 & 99.99 & $22^{\circ} \mathrm{S}-23^{\circ} \mathrm{S}$ & 21,22 \\
\hline \multirow[t]{2}{*}{$S_{2}$} & 429.9 & 26.9 & 1.1 & 99.99 & $16^{\circ} \mathrm{S}$ & 22 \\
\hline & 429.9 & 26.9 & 0.7 & 99.90 & $19^{\circ} \mathrm{S}-21^{\circ} \mathrm{S}$ & 23 \\
\hline \multirow[t]{2}{*}{$\mathrm{S}_{3}$} & 434.3 & 26.7 & 1.2 & 99.99 & $10^{\circ} \mathrm{S}-12^{\circ} \mathrm{S}$ & 23 \\
\hline & 437.6 & 26.5 & 0.6 & 99.90 & $11^{\circ} \mathrm{S}$ & 23 \\
\hline \multirow[t]{3}{*}{$\mathrm{T}_{1}$} & 426.6 & 27.1 & 1.1 & 99.99 & $23^{\circ} \mathrm{N}-25^{\circ} \mathrm{N}$ & 22 \\
\hline & 429.9 & 26.9 & 0.8 & 99.99 & $11^{\circ} \mathrm{N}-12^{\circ} \mathrm{N}$ & 21,22 \\
\hline & 429.9 & 26.9 & 0.9 & 99.90 & $16^{\circ} \mathrm{N}-20^{\circ} \mathrm{N}$ & 21,22 \\
\hline \multirow[t]{2}{*}{$\mathrm{T}_{2}$} & 433.2 & 26.7 & 0.6 & 99.99 & $6^{\circ} \mathrm{N}-8^{\circ} \mathrm{N}$ & 21,22 \\
\hline & 433.2 & 26.7 & 0.9 & 99.99 & $11^{\circ} \mathrm{N}-12^{\circ} \mathrm{N}$ & 21,22 \\
\hline \multirow[t]{2}{*}{$\mathrm{T}_{3}$} & 435.4 & 26.6 & 0.9 & 99.90 & $14^{\circ} \mathrm{N}-15^{\circ} \mathrm{N}$ & 23 \\
\hline & 437.6 & 26.4 & 1.0 & 99.99 & $16^{\circ} \mathrm{N}$ & $21,22,23$ \\
\hline $\mathrm{U}$ & 463.1 & 25.0 & 0.5 & 99.00 & $14^{\circ} \mathrm{S}$ & 22 \\
\hline $\mathrm{M}^{\prime}$ & 398.9 & 29.0 & 2.3 & 99.90 & $16^{\circ} \mathrm{N}-18^{\circ} \mathrm{N}$ & 22 \\
\hline \multirow[t]{2}{*}{$\mathrm{R}^{\prime}$} & 409.9 & 28.2 & 7.1 & 99.99 & $13^{\circ} \mathrm{S}-19^{\circ} \mathrm{S}$ & 21,22 \\
\hline & 413.3 & 28.0 & 6.9 & 99.99 & $13^{\circ} \mathrm{S}-18^{\circ} \mathrm{S}$ & 21,22 \\
\hline \multirow[t]{2}{*}{$S_{1}^{\prime}$} & 423.2 & 27.3 & 2.8 & 99.99 & $9^{\circ} \mathrm{S}-12^{\circ} \mathrm{S}$ & 21,23 \\
\hline & 425.5 & 27.2 & 2.6 & 99.90 & $8^{\circ} \mathrm{S}-13^{\circ} \mathrm{S}$ & 23 \\
\hline \multirow[t]{2}{*}{$S_{2}^{\prime}$} & 428.8 & 27.0 & 2.7 & 99.99 & $9^{\circ} \mathrm{S}-10^{\circ} \mathrm{S}$ & 22 \\
\hline & 432.1 & 26.8 & 3.0 & 99.99 & $11^{\circ} \mathrm{S}-12^{\circ} \mathrm{S}$ & 22 \\
\hline $\mathrm{S}_{3}^{\prime}$ & 435.4 & 26.6 & 3.6 & 99.99 & $11^{\circ} \mathrm{S}-13^{\circ} \mathrm{S}$ & 23 \\
\hline$S_{4}^{\prime}$ & 441.0 & 26.2 & 3.0 & 99.99 & $16^{\circ} \mathrm{S}-17^{\circ} \mathrm{S}$ & 22 \\
\hline \multirow[t]{3}{*}{$\mathrm{T}_{1}^{\prime}$} & 425.5 & 27.2 & 1.2 & 99.90 & $26^{\circ} \mathrm{N}-41^{\circ} \mathrm{N}$ & 22 \\
\hline & 427.7 & 27.1 & 2.6 & 99.99 & $18^{\circ} \mathrm{N}-20^{\circ} \mathrm{N}$ & 22 \\
\hline & 429.9 & 27.1 & 2.0 & 99.99 & $23^{\circ} \mathrm{N}-25^{\circ} \mathrm{N}$ & 21 \\
\hline $\mathrm{T}_{2}^{\prime}$ & 433.2 & 26.7 & 3.8 & 99.99 & $15^{\circ} \mathrm{N}-25^{\circ} \mathrm{N}$ & 21 \\
\hline $\mathrm{T}_{3}^{\prime}$ & 436.5 & 26.5 & 3.5 & 99.99 & $13^{\circ} \mathrm{N}-18^{\circ} \mathrm{N}$ & 23 \\
\hline $\mathrm{U}_{1}^{\prime}$ & 455.4 & 25.4 & 2.6 & 99.90 & $13^{\circ} \mathrm{S}-16^{\circ} \mathrm{S}$ & 22 \\
\hline $\mathrm{U}_{2}^{\prime}$ & 462.0 & 25.1 & 3.3 & 99.99 & $16^{\circ} \mathrm{S}-17^{\circ} \mathrm{S}$ & 21,23 \\
\hline
\end{tabular}

differential rotation laws as derived from Greenwich sunspot data (Balthasar et al. 1986) and Mt. Wilson Doppler shifts (Snodgrass 1984), respectively, are also shown. Please note that all rotation frequencies and periods are synodic (unless otherwise stated).

The corresponding diagrams for each separate cycle are displayed in Figs. 8-10. Dotted contour lines here indicate the $99.9 \%$ significance level $\left(P_{p=0.999}\right.$, Figs. $\left.2 \mathrm{c}-2 \mathrm{~h}\right)$. The same labels as in Figs. 7a and $7 \mathrm{~b}$ have been used for peaks at similar frequencies, and the cycle number has been added as an additional subscript. Labels in lower case indicate peaks that 

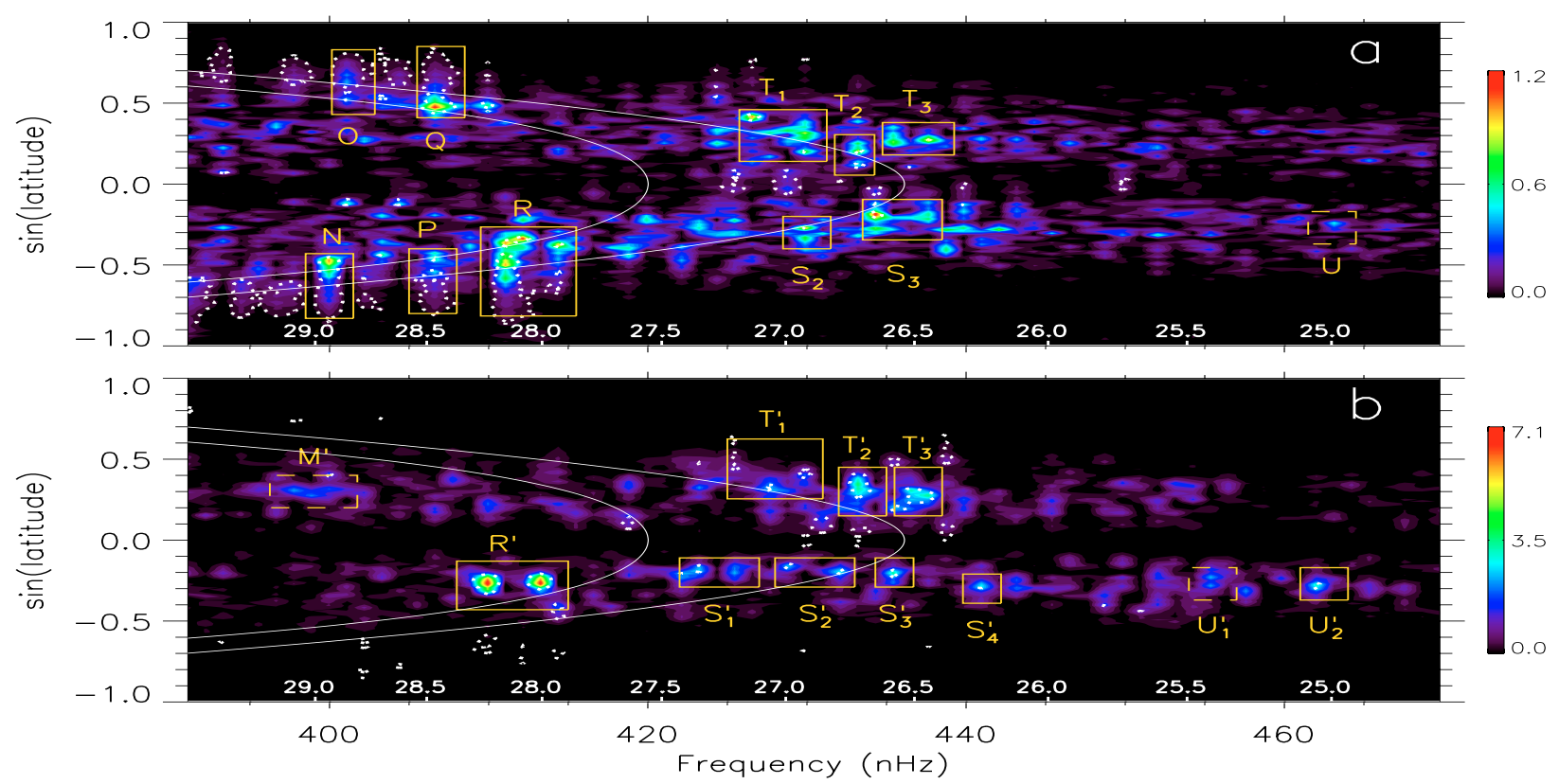

Fig. 7. a) FFT power density (in arbitrary but constant units) vs. sine latitude and frequency for the net (signed) magnetic flux. The frequency range is $390-470 \mathrm{nHz}$ (corresponding to periods $29.7-24.6 \mathrm{~d}$, indicated in white on the horizontal axis). Dotted contour lines represent the 99.99\% significance level. The labeled regions are listed in Table 3. The two curves indicate the differential rotation laws for sunspots (right curve) and Doppler shifts (left curve). b) The same for the total (unsigned) magnetic flux.

are present in a specific cycle but cannot be clearly associated with a corresponding peak in Figs. 7a or 7b. The latitudefrequency diagrams for the second harmonic frequency range $780 \mathrm{nHz} \leq v \leq 940 \mathrm{nHz}\left(14.84 \mathrm{~d} \geq v^{-1} \geq 12.31 \mathrm{~d}\right)$ are also shown. This allows us to identify two or four sector patterns in the large scale magnetic field ( $m=1$ or 2 , in terms of the azimuthal order). All labeled peaks are listed in Table 4 for cycle 21, Table 5 for cycle 22, and Table 6 for cycle 23 .

A common feature of all the latitude-frequency diagrams in the fundamental frequency range (Figs. 7a,b-10a,b) is that the spectral power above the noise level is not uniformly distributed along the differential rotation curves, as one might expect, but rather concentrated in a few distinct regions. These regions with significant power often appear at well defined frequencies but can be quite wide in latitude, which indicates that the large-scale magnetic field structures must be sufficiently coherent and long-lived to concentrate significant power only at certain narrow frequency intervals. In addition, there is a distinct asymmetry between the northern and southern hemispheres, i.e. regions having significant power are not symmetric with respect to the equator. These properties have already been noted by Antonucci et al. (1990), who performed a similar analysis for the net magnetic flux during cycle 21 .

In the following, we first discuss the results for the southern hemisphere and then continue with the northern hemisphere.

\subsection{Rotation frequencies in the southern hemisphere}

The dominant feature in the southern part $(\sin \vartheta<0)$ of the latitude-frequency diagram of the total magnetic flux (Fig. 7b) is the very distinct double peak $\mathrm{R}^{\prime}$ at $v_{1}=409.9 \pm 0.6 \mathrm{nHz}$ $(28.24 \pm 0.04 \mathrm{~d})$ and $v_{2}=413.3 \pm 0.6 \mathrm{nHz}(28.00 \pm 0.04 \mathrm{~d})$.
The latitudinal extent is approximately $10^{\circ}-20^{\circ} \mathrm{S}$. The corresponding double peak $\mathrm{R}$ in the net magnetic flux (Fig. 7a) appears at slightly shifted frequencies and covers latitudes $\sim 20^{\circ}-60^{\circ} \mathrm{S}$. A northern analogue does not exist, neither in the total nor in the net flux. The comparison with the latitudefrequency diagrams of the separate cycles shows that this peak appeared not only in cycle 21 but also in cycle 22 (regions $R_{21}$ and $\mathrm{R}_{21}^{\prime}$ in Figs. $8 \mathrm{a}$ and $8 \mathrm{~b}$, regions $\mathrm{R}_{22}$ and $\mathrm{R}_{22}^{\prime}$ in Figs. 9a and $9 \mathrm{~b}$ ). This indicates that during both cycles 21 and 22 , the large-scale southern magnetic field pattern rotated nearly rigidly with the period 28.0-28.2 d. During cycle 23, less power at slightly higher frequencies $(415.3-419.8 \mathrm{nHz})$ is seen in the total flux (peak $R_{23}^{\prime}$ in Fig. 10b) but not in the net flux (Fig. 10a). Interestingly, Bai (1990) reported a "hot spot" with a rotation period of $27.99 \mathrm{~d}$ in the southern hemisphere for cycles 19-22. The term "hot spot" refers to a region of enhanced flare activity. We will return to this point in the following section.

The less dominant southern peaks $S_{1}^{\prime}-S_{4}^{\prime}$ in the total flux (Fig. 7b) have frequencies of $423.2-425.5 \mathrm{nHz}$, $428.8-432.1 \mathrm{nHz}, 435.4 \mathrm{nHz}$, and $441.0 \mathrm{nHz}$ (corresponding to periods of 27.3-27.2 d, 27.0-26.8 d, $26.6 \mathrm{~d}$, and $26.2 \mathrm{~d}$ ). In the net flux, only peaks $S_{2}$ and $S_{3}$ reach the $99.99 \%$ significance level. The latitude-frequency diagrams of the separate cycles show that peak $S_{1}^{\prime}$ occurred in cycle 21 (cf. $S_{1,21}^{\prime}$ in Fig. 8b), with similar power as peak $R_{21}^{\prime}$, and again in cycle $23\left(S_{1,23}^{\prime}\right.$ in Fig. 10b), where it is even the strongest peak in the southern hemisphere. During cycle 22, there are significant but scattered peaks at similar frequencies in the net flux $\left(S_{1,22}\right.$ in Fig. 9a) but not in the total flux (Fig. 9b). However, the unsigned as well as the signed flux show significant power at the second harmonic (regions $S_{1,22}^{*}$ in Fig. 9c and $S_{1,22}^{*}$ in Fig. 9d). Note 
that the peaks at the second harmonic in the net flux show approximately twice as much power as the fundamental peaks. This may possibly be interpreted as the pattern of two activity complexes separated by $180^{\circ}$ in longitude and rotating with the same frequency (see the discussion in the following section). The reason why the fundamental frequency does not appear in the total flux may be due to the circumstance that an oscillation that alternates between plus and minus in the net flux has approximately the doubled frequency in the total flux, as is the case for the $22 \mathrm{yr}$ magnetic and $11 \mathrm{yr}$ sunspot cycle (cf. Figs. 3a,b). The peaks $S_{2}^{\prime}$ and $S_{4}^{\prime}$ in Fig. $7 b$ are probably due to the corresponding peaks in cycle $22\left(S_{2,22}^{\prime}\right.$ and $S_{4,22}^{\prime}$ in Fig. 9b), while the peak $S_{3}^{\prime}$ was formed during cycle $23\left(S_{3,23}^{\prime 2}\right.$ in Fig. 10b). Note, however, that because of the reduced frequency resolution of the latitude-frequency diagrams of cycle 22 and especially cycle 23 , it is difficult to decide from which cycle the main contributions to $S_{2}^{\prime}$ and $S_{3}^{\prime}$ in Fig. $7 b$ actually come.

Surprisingly, two significant peaks occur at very high frequencies in the latitude-frequency diagram of the total flux in Fig. 7b, namely peak $\mathrm{U}_{1}^{\prime}$ at $v=455.4 \pm 0.6 \mathrm{nHz}(25.42 \pm$ $0.03 \mathrm{~d})$ and $\mathrm{U}_{2}^{\prime}$ at $v=462.0 \pm 0.6 \mathrm{nHz}(25.05 \pm 0.03 \mathrm{~d})$. Both peaks are located in the southern hemisphere. The corresponding peak $U$ in the signed flux is rather weak. It is clearly seen from the diagrams for the separate cycles that peak $\mathrm{U}_{2}^{\prime}$ was present during cycles 21 and 23 , while peak $U_{1}^{\prime}$ occurred during cycle 22 (cf. $\mathrm{U}_{2,21}^{\prime}$ in Fig. 8b, $\mathrm{U}_{1,22}^{\prime}$ in Fig. 9b, and $\mathrm{U}_{2,23}^{\prime}$ in Fig. 10b). These regions appear at much higher frequencies than one would expect from the differential rotation laws (the synodic rotation rate for sunspots at these latitudes is approximately $430 \mathrm{nHz}$ ). The circumstance that there is significant power at a synodic frequency of approximately $460 \mathrm{nHz}$ for all three cycles makes it unlikely that these peaks are due to random fluctuations. Interestingly, the sidereal rotation rate of the equator is approximately $460 \mathrm{nHz}$. A possible explanation for these peaks will be discussed in Sect. 6 .

The wavelet power spectrum of the total magnetic flux averaged over latitudes $10^{\circ} \mathrm{S}-20^{\circ} \mathrm{S}$ is displayed in Fig. 11b. Clearly visible is the $\sim 28 \mathrm{~d}$ periodicity during cycles 21 and 22 . Less pronounced is the $\sim 27.3 \mathrm{~d}$ periodicity during cycle 23 . Significant power can also be seen for the 25.0-25.5 d periodicity during all three cycles, in particular during cycle 22 .

\subsection{Rotation frequencies in the northern hemisphere}

In the northern part $(\sin \vartheta>0)$ of the latitude-frequency diagram of the total flux (Fig. 7b), two distinct peaks $\mathrm{T}_{2}^{\prime}$ and $\mathrm{T}_{3}^{\prime}$ appear at $v=433.2 \pm 0.6 \mathrm{nHz}(26.72 \pm 0.04 \mathrm{~d})$ and $v=436.5 \pm$ $0.6 \mathrm{nHz}(26.52 \pm 0.04 \mathrm{~d})$, respectively. Less dominant peaks can be seen in the region $\mathrm{T}_{1}^{\prime}$ at frequencies $425.5-429.9 \mathrm{nHz}$ (27.20-26.92 d) and in $\mathrm{M}^{\prime}$ at $398.9 \pm 0.6 \mathrm{nHz}(29.0 \pm 0.04 \mathrm{~d})$. Figure $7 \mathrm{a}$ for the net flux shows a similar picture for regions $\mathrm{T}_{1}-\mathrm{T}_{3}$, but no significant analogue to peak $\mathrm{M}^{\prime}$ is present. Similarly, peaks $\mathrm{O}$ at $v=401.1 \pm 0.6 \mathrm{nHz}(28.86 \pm 0.04 \mathrm{~d})$ and $\mathrm{Q}$ at $v=406.6 \pm 0.6 \mathrm{nHz}(28.47 \pm 0.04 \mathrm{~d})$ in Fig. 7a have no significant analogue in the total flux in Fig. $7 \mathrm{~b}$.

The latitude-frequency diagrams of the separate cycles show that peak $\mathrm{T}_{2,21}^{\prime}$ at frequencies $430.5-433.6 \mathrm{nHz}$

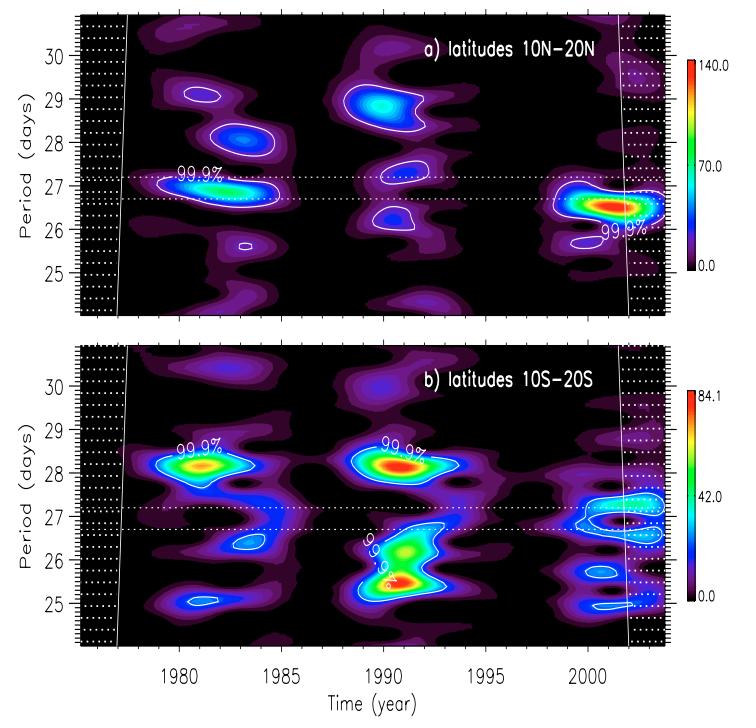

Fig. 11. a) Wavelet power spectrum of the total magnetic flux averaged over latitudes $10^{\circ} \mathrm{N}-20^{\circ} \mathrm{N}$. Morlet wavelets with a nondimensional frequency of 120 were used to achieve a maximum frequency resolution. White contours indicate the $99.9 \%$ significance level, hatched regions the cone of influence. The horizontal dashed lines represent the synodic rotation periods of sunspots at $10^{\circ} \mathrm{N}$ and $20^{\circ} \mathrm{N}$, respectively. During cycle 21 , most power is concentrated around a periodicity of $27.0 \mathrm{~d}$, while cycle 22 shows dominant power around $29.0 \mathrm{~d}$. During cycle 23 , most power is concentrated around the periodicity of $26.4 \mathrm{~d}$. b) The same for latitudes $10^{\circ} \mathrm{S}-20^{\circ} \mathrm{S}$. The $28.0 \mathrm{~d}$ period is prominent for cycles 21 and 22, while the 25.0-25.5 d period occurs during all three cycles and is particularly pronounced during cycle 22 .

(26.9-26.7 d) is the dominating northern feature in the total flux during cycle 21 (Fig. 8b). There is also a peak at the second harmonic $\left(\mathrm{T}_{2,21}^{\prime *}\right.$ in Fig. 8d). The same frequencies are seen for the net flux (peaks $\mathrm{T}_{2,21}$ in Fig. 8a and $\mathrm{T}_{2,21}^{*}$ in Fig. 8c), which was already noted by Antonucci et al. (1990) and interpreted as a four sector pattern $(m=2)$ in the large-scale magnetic field. However, the classification of such patterns in terms of the azimuthal order $m$ should be used with caution. By definition, a $m=2$ structure corresponds to four sectors (each $90^{\circ}$ wide in longitude) that have equal amplitudes but opposite polarities. A different configuration, for instance two large bipolar active regions rotating with the same period but separated by $180^{\circ}$ in longitude, may also cause a peak at the fundamental rotation frequency and its second harmonic. Interestingly, Bai (2003a) reported a "double-hot-spot" system with a rotation period of $26.73 \mathrm{~d}$ that operated in the northern hemisphere during cycles 20 and 21. As already mentioned, the term "hot spot" refers to a region of enhanced flare activity and is used by Bai (2003a) instead of "active longitude" to emphasize that the longitude distributions of flares are different in the two hemispheres. A double-hot-spot system consists of two hot spots that are separated by $180^{\circ}$ in longitude and rotate with the same period. Since the periodicities coincide, it is reasonable to conclude that the large-scale magnetic pattern that caused peaks $\mathrm{T}_{2,21}^{\prime}$ and $\mathrm{T}_{2,21}^{\prime *}$ in Figs. $8 \mathrm{~b}$ and $8 \mathrm{~d}$ was related with the double-hotspot system during cycle 21 . Similar structures of two activity centers separated by $180^{\circ}$ were found in sunspot activity by 
Berdyugina \& Usoskin (2003) in both north and south. An average rotation period of these structures for the last 120 years is $26.9 \mathrm{~d}$ which corresponds to the mean long-term latitude of sunspot formation of $14^{\circ}$.

The following cycle 22 exhibits increased power at similar frequencies, i.e. peak $\mathrm{T}_{1,22}^{\prime}$ in the unsigned flux (Fig. 9b) at $424.3 \mathrm{nHz}\left(27.3 \mathrm{~d}\right.$ ) and peak $\mathrm{T}_{1,22}$ in the signed flux (Fig. 9b) at approximately the same frequency. However, there is more power in the unsigned flux in region $\mathrm{M}_{22}^{\prime}$ at $398.6 \mathrm{nHz}(29.0 \mathrm{~d})$, while for the signed flux it is region $\mathrm{Q}_{22}$ at $398.6-408.3 \mathrm{nHz}$ (29.0-28.3 d) that dominates. During cycle 23, the dominant northern peak is $\mathrm{T}_{3,23}^{\prime}$ at $437.9 \mathrm{nHz}(26.4 \mathrm{~d})$ in the unsigned flux and $\mathrm{T}_{3,23}$ at the same frequency in the signed flux.

An overview is given in Fig. 11a, which displays the wavelet power spectrum of the total magnetic flux averaged over latitudes $10^{\circ} \mathrm{N}-20^{\circ} \mathrm{N}$. The periodicity around $27.0 \mathrm{~d}$ was particularly pronounced during cycle 21 , while the $29.0 \mathrm{~d}$ periodicity was more prominent during cycle 22 . Finally, cycle 23 shows most power at the $26.4 \mathrm{~d}$ periodicity.

Active regions strongly tend to emerge near or within already existing active regions, forming so-called "complexes of activity" or "active/preferred longitudes" (e.g. Trotter \& Billings 1962; Bumba \& Howard 1969; Bogart 1982; Gaizauskas et al. 1983; Brouwer \& Zwaan 1990; Gaizauskas et al. 2001). These complexes are maintained by emergence of new magnetic flux and may persist from 3-6 to 20-40 consecutive solar rotations. We assume that the above described features of the rotation of large-scale magnetic fields are closely linked to such activity complexes. Neugebauer et al. (2000) reported a $27.0 \mathrm{~d}$ periodicity in the solar wind speed and the radial component of the interplanetary magnetic field from 1962 to 1998 and attributed it to the existence of preferred longitudes. The authors noted that the $27.0 \mathrm{~d}$ periodicity can be discerned only over long periods of time and that it is less apparent or absent when the data are analyzed for time intervals of one solar cycle or less. Ruzmaikin et al. (2001) detected the same periodicity using synoptic charts of the photospheric magnetic field recorded at the Wilcox Solar Observatory from 1976 to 2000 . Henney \& Harvey (2002) found the $27.0 \mathrm{~d}$ periodicity also in the NSO/KP synoptic maps (1977-2001) and concluded that it was coherent over two decades and due to long-lived complexes of active regions in the northern hemisphere at a latitude of $\sim 18^{\circ}$. A phase coherence analysis of the international sunspot number showed that the $27.0 \mathrm{~d}$ period lacked a clear coherence during the interval 1880-1977.

\section{Possible $r$-mode signatures in the magnetic flux}

In the previous sections, we have shown that the magnetic flux exhibits a multitude of quasi-periodic oscillations. There have recently been suggestions by Sturrock et al. (1999), Lou (2000) and Sturrock (2004) that the Rieger and similar Rieger-type periodicities are related to physical properties of Rossby-type waves, which are often referred to as $r$-modes (Papaloizou \& Pringle 1978; Provost et al. 1981; Smeyers et al. 1981; Saio 1982; Wolff \& Blizard 1986; Wolff 1998, 2000; Lou 2000; Dzhalilov et al. 2002; Dzhalilov \& Staude 2004). We will now consider the possibility that $r$-modes are the cause for the periodicities in the magnetic flux.

The $r$-modes are globally coherent quasi-toroidal oscillations dominated by the Coriolis force, which in the limit of small scales reduce to the Rossby waves in geophysics (Provost et al. 1981). Rossby-type waves belong to a subset of global tidal waves described by Laplace's tidal equation. They can exist in a fluid layer on the surface of a rotating sphere and can give rise to detectable features such as large-scale velocity patterns and surface elevations (Lou 2000). For instance, Rossby waves have been observed in the terrestrial oceans as largescale variations of sea-surface height with amplitudes $\leq 10 \mathrm{~cm}$ and wavelengths $\geq 500 \mathrm{~km}$ (Chelton \& Schlax 1996). Kuhn et al. (2000) have reported the detection of $100 \mathrm{~m}$ high "hills" in the solar photosphere, spaced uniformly over the solar surface with a characteristic separation of approximately $90000 \mathrm{~km}$ (corresponding to $m \approx 50$ ). These observations were made with the Michelson Doppler Imager (MDI) on board the Solar and Heliospheric Observatory (SOHO) and interpreted as the photospheric signature of long-period $r$-modes. Ulrich (2001) reported long-lived torsional wave patterns in the solar surface velocity field which resemble $r$-modes.

The $r$-modes are retrograde waves that can be classified using the usual spherical harmonic indices $(l, m, n)$, where $l$ and $m$ are the degree and azimuthal order, respectively, and $n$ is the radial order. In a uniformly rotating sphere with the sidereal rotation frequency $v_{\mathrm{S}}$, the $r$-modes have rotation frequencies $-2 v_{\mathrm{S}} / l(l+1)$ in the corotating system (negative since the waves are retrograde). The radial fine splitting, which lifts the degeneracy in $n$, is small for $n \leq 14$ (Wolff \& Blizard 1986). The degeneracy in $m$ is lifted if differential rotation of the stellar envelope is taken into account, and certain modes may become prohibited (Wolff 1998). However, most rates for the real Sun would probably differ only by a few percent $(\leq 10 \mathrm{nHz})$ from the case of no differential rotation.

For a rigidly rotating sphere, a corotating observer would detect oscillations at the frequencies

$v_{l, m, \mathrm{rot}}=\frac{2 m v_{\mathrm{S}}}{l(l+1)}$,

where the negative sign due to the retrograde motion has been disregarded. If we use $v_{\mathrm{S}}=455 \mathrm{nHz}$ for the (sidereal) equatorial rotation frequency of the Sun (Schou et al. 1998), an observer on Earth would expect to see the frequencies

$v_{l, m, \text { syn }}=m\left(v_{\mathrm{S}}-v_{\mathrm{E}}\right)-v_{l, m, \mathrm{rot}}$,

where $v_{\mathrm{E}}=1 \mathrm{yr}^{-1}=31.7 \mathrm{nHz}$. However, if the $r$-modes interact with and modulate the magnetic field pattern that corotates with the Sun, then we would expect to find the frequencies $v_{l, m, \text { rot }}$ rather than $v_{l, m, \text { syn }}$ in the magnetic data (cf. Sturrock 2004, and references therein).

A list of $r$-mode frequencies is given for $l=1, \ldots, 10$ and $m=1, l$ in Table 7 . For comparison, the frequencies derived by Lou (2000) for equatorially trapped Rossby waves in the corotating frame are also listed. These frequencies depend only on $m$ and $n$. Eq. (13) in Lou (2000) gives for the case $n=1$ :

$v_{m, \text { rot }}=v_{\mathrm{S}}\left[\frac{|m|}{2}+0.172 \frac{3}{|m|}\right]^{-1}$. 
Table 7. Frequency estimates for $r$-modes (Eqs. (3) and (4)) and equatorially trapped Rossby waves (Eq. (5)), calculated with $v_{\mathrm{S}}=455 \mathrm{nHz}$.

\begin{tabular}{rrrr|rr|rr}
\hline \hline$l$ & $m$ & $v_{l, m, \text { syn }}$ & $v_{l, m, \text { rot }}$ & $m$ & $v_{m, \text { rot }}$ & $m$ & $v_{m, \text { rot }}$ \\
\hline 1 & 1 & -31.7 & 455 & 1 & 448 & 20 & 45.4 \\
2 & 1 & 272 & 152 & 2 & 362 & 21 & 43.2 \\
2 & 2 & 543 & 303 & 3 & 272 & 22 & 41.3 \\
3 & 1 & 347 & 75.8 & 4 & 214 & 23 & 39.5 \\
3 & 3 & 1042 & 228 & 5 & 175 & 24 & 37.8 \\
4 & 1 & 378 & 45.5 & 6 & 147 & 25 & 36.3 \\
4 & 4 & 1511 & 182 & 7 & 127 & 26 & 34.9 \\
5 & 1 & 393 & 30.3 & 8 & 112 & 27 & 33.7 \\
5 & 5 & 1965 & 152 & 9 & 100 & 28 & 32.5 \\
6 & 1 & 402 & 21.7 & 10 & 90.1 & 29 & 31.3 \\
6 & 6 & 2410 & 130 & 11 & 82.0 & 30 & 30.3 \\
7 & 1 & 407 & 16.3 & 12 & 75.3 & 31 & 29.3 \\
7 & 7 & 2849 & 114 & 13 & 69.6 & 32 & 28.4 \\
8 & 1 & 411 & 12.6 & 14 & 64.7 & 33 & 27.5 \\
8 & 8 & 3285 & 101 & 15 & 60.4 & 34 & 26.7 \\
9 & 1 & 413 & 10.1 & 16 & 56.6 & 35 & 26.0 \\
9 & 9 & 3718 & 91. & 17 & 53.3 & 36 & 25.3 \\
10 & 1 & 415 & 8.27 & 18 & 50.4 & 37 & 24.6 \\
10 & 10 & 4150 & 82.7 & 19 & 47.8 & 38 & 23.9 \\
\hline & & & & &
\end{tabular}

Note that if $m$ is sufficiently large, we have $v_{m, \text { rot }} \approx 2 v_{\mathrm{S}} / m$ for equatorially trapped Rossby waves and $v_{m, m, \text { rot }}=2 v_{\mathrm{S}} /(m+1) \approx$ $v_{m \text {,rot }}$ for sectoral $r$-modes (i.e. $l=|m|>0$ ). These are also the modes which are least affected by differential rotation, since their motions are concentrated most strongly toward the equator. For conceptual aspects of possible interactions of equatorially trapped Rossby waves with the magnetic field in the photosphere see Lou et al. (2003).

Lou (2000) has pointed out that for even $m=4,6,8,10$, and 12, the period estimates $v_{m \text {,rot }}^{-1}=54,79,103,128$ and $154 \mathrm{~d}$ for Rossby waves are in good agreement with the observed Rieger and Rieger-type periodicities. In Sect. 4, we have shown that the total magnetic flux exhibits significant power at the frequencies 93.1-89.7, 76.5-73.1, 65.4, 55.4-52.1, 49.9-46.5, 41.0 , and $37.7-34.3 \mathrm{nHz}$ (cf. Table 2), which is very close to the frequency estimates $v_{m \text {,rot }}$ for $m=10,12,14,16,18,20,22,24$, and 26 (cf. Table 7). Can this series be extended to even higher values of $m$ ? As already mentioned, Kuhn et al. (2000) have reported a possible long-period $r$-mode signature in the photosphere that would correspond to $m \approx 50$. The frequency estimate for this $r$-mode is $v_{m=50 \text {,rot }}=18.2 \mathrm{nHz}(1.74 \mathrm{yr})$. Indeed, the total magnetic flux in the southern hemisphere exhibits significant power in the frequency range $17.7-18.8 \mathrm{nHz}$ (cf. region $\mathrm{B}_{2}^{\prime}$ in Fig. $3 \mathrm{~b}$ and Table 1). The corresponding time interval $\sim 1996.5-2003.0$ overlaps with the MDI data set (April 1996-June 1998) used by Kuhn et al. (2000). In Paper I, we have shown that the photospheric flux excess gradually changed from the southern to the northern hemisphere from 1995 to 2001 and that it was modulated by a $1.79 \pm 0.06$ yr periodicity. Thus, if the $1.7-1.8 \mathrm{yr}$ periodicity in the total magnetic flux is really due to $r$-modes, it is possible that these modes also play a role in the north-south asymmetry of the photospheric magnetic flux.

Empirically, the frequencies in the total magnetic flux as observed from Earth agree well with the frequency estimates for equatorially trapped Rossby waves (or sectoral $r$-modes) in the corotating frame of the Sun. However, the observed frequencies might as well be due to tesseral $(0<|m|<l)$ $r$-modes (Sturrock et al. 1999; Sturrock 2004). The Rieger period at $73.1-76.5 \mathrm{nHz}$ could be related to the $r$-mode with $l=3, m=1$, which has frequencies $v_{l=3, m=1, \text { rot }}=76 \mathrm{nHz}$ and $v_{l=3, m=1, \text { syn }}=347 \mathrm{nHz}$. We indeed find increased power around $350 \mathrm{nHz}$ in the total and net magnetic flux at latitude $\sim 11^{\circ} \mathrm{S}$, but at a rather low significance level of $95 \%$. Similarly, the double peak $\mathrm{K}^{\prime}$ at $93.1-89.7 \mathrm{nHz}$ in Fig. 5 b could be due to the $r$-mode with $l=4, m=2$, which has frequencies $v_{l=4, m=2, \text { rot }}=91 \mathrm{nHz}$ and $v_{l=4, m=2, \text { syn }}=756 \mathrm{nHz}$. Again, we find power at the $95 \%$ significance level in the range $765-775 \mathrm{nHz}$. In this manner, many if not all of the periodicities detected in the magnetic flux could be assigned to tesseral $r$-modes. On the other hand, Wolff (1998) has shown that the differential rotation on the solar surface tends to prohibit certain tesseral modes (including the modes with $(l, m)=(3,1)$ and $(4,2))$, whereas all sectoral modes with $l=|m|>1$ would survive. Therefore, if the Rieger and Rieger-type frequencies are related to tesseral $r$-modes, these modes would need to be confined to a region in the solar interior with approximately rigid rotation. Such a region might be at the bottom of the convection zone, where the solar dynamo is assumed to be located. However, if the surface elevations observed by Kuhn et al. (2000) are real and due to $r$-mode oscillations, this would instead imply that sectoral $r$-modes (or equatorially trapped Rossby waves) in the surface layer are more likely to be the cause of the periodicities detected in the magnetic flux.

Is there also evidence for possible $r$-mode signatures in the high frequency range of the total magnetic flux? Equatorially trapped Rossby waves with $m=1$ have the estimated frequency $v_{m \text {,rot }}=448 \mathrm{nHz}, r$-modes with $l=|m|=1$ have $v_{l, m, \text { rot }}=455 \mathrm{nHz}$. We have shown in the previous section that we find significant peaks around $\sim 455-465 \mathrm{nHz}$ (synodic) in the southern hemisphere during all three cycles 21-23 (cf. Figs. 7b-11b, Tables 3-6). Since these peaks are quite far away from the rotation frequencies of magnetic active regions, they could also be due to equatorially trapped Rossby waves or $r$-modes.

\section{Conclusions}

The present time series analysis of NSO/Kitt Peak synoptic maps of the photospheric magnetic field from 1975 to 2003 has revealed a multitude of (quasi-) periodic oscillations in the range from $25 \mathrm{~d}$ to $22 \mathrm{yr}$, which are listed in Tables 1 to 6 .

In the range between $1 \mathrm{yr}$ and $22 \mathrm{yr}$ (cf. Sect. 3), we have located the sources for the $1.5 \mathrm{yr}, 1.8 \mathrm{yr}$, and $3.6 \mathrm{yr}$ periodicities that were previously detected in the north-south asymmetry of the total magnetic flux for the time intervals 1978-1984, 1984-1995, and 1995-2001, respectively (Knaack et al. 2004). The $1.5 \mathrm{yr}$ period is due to the combination of a $1.4 \mathrm{yr}$ and 
a $1.6 \mathrm{yr}$ periodicity that occurred in the total flux at latitudes $\sim 10^{\circ}-16^{\circ}$ north and south, respectively, from 1977 to 1985 . This is in agreement with the analysis of $\mathrm{H} \alpha$ flares by Ichimoto et al. (1985), who noted that two periodicities of $\sim 1.5 \mathrm{yr}$ occurred in anti phase in the two hemispheres during cycle 21 . The $1.8 \mathrm{yr}$ period has been located only in the south at latitudes $\sim 20^{\circ}-25^{\circ}$. It occurred in the total flux mainly from 1997 to 2003 and may be related to a possible $r$-mode signature in the photosphere with azimuthal order $m \approx 50$ reported by Kuhn et al. (2000). The period estimate for such an $r$-mode is close to $1.8 \mathrm{yr}$.

The $\sim 3.6 \mathrm{yr}$ period has been detected in the total flux in both hemispheres, overlapping each other during 1987-1992. These oscillations may be linked to the $3.65 \mathrm{yr}$ period in the south and $3.8 \mathrm{yr}$ period in the north reported by Berdyugina \& Usoskin (2003) in their analysis of active longitudes in sunspot activity over the last 120 years. In addition, we have found evidence for periodicities around $2.6 \mathrm{yr}$ and $1.3 \mathrm{yr}$. The latter appeared at intermediate latitudes $\sim 30^{\circ}-55^{\circ}$ in both hemispheres. It was present during all three cycles $21-23$ and particularly prominent during cycle 22 . This $1.3 \mathrm{yr}$ period appears to be related to large-scale magnetic surges toward the poles (e.g. Wang et al. 1989), which emanate at these latitudes. These surges may also be the cause for the 1.3-1.4 yr periodicities reported in the interplanetary magnetic field and geomagnetic activity (Lockwood 2001, and references therein) as well as in sunspot areas and sunspot numbers (Krivova \& Solanki 2002). Whether or not the $1.3 \mathrm{yr}$ periodicity in the total magnetic flux is related to the $1.3 \mathrm{yr}$ periodicity detected by Howe et al. (2000) in the rotation rate at the base of the convection zone is an open question.

Periodicities in the range between 100 and 350 days (cf. Sect. 4) have been detected in the total magnetic flux of the southern hemisphere at 124-129 d, 151-158 d, $177 \mathrm{~d}$, 209-222 d, 232-249 d, $282 \pm 4 \mathrm{~d}$ and 307-337 d. The net flux shows an additional periodicity of 100-103 d. These periodicities agree very well with period estimates for equatorially trapped Rossby waves with $m=8,10,12, \ldots, 26$ (cf. Sect. 6). We have found evidence that the Rieger periodicity at $155 \mathrm{~d}$ was present in the magnetic flux not only during cycle 21 but also during cycle 22 , in contrast to a study by Ballester et al. (2002). Preliminary results indicate that it has occurred again in cycle 23, in agreement with Ballester et al. (2004). However, the question of whether the Rieger period was present during a specific cycle or not seems somewhat secondary as compared to the circumstance that it is one periodicity amongst a multitude of other, similarly important ones.

As far as the rotation of large-scale photospheric magnetic fields during cycles $21-23$ is concerned, we can confirm the conclusions by Antonucci et al. (1990). Rotational power is not uniformly distributed along the differential rotation curve for sunspots, but concentrated in a few distinct regions at discrete frequencies, which can extend considerably over wide latitude zones. These regions are usually not symmetric with respect to the equator. The (independent) evolution of long-lived activity complexes in both hemispheres may be the cause for this behavior.
We have found that the pronounced $28.1 \mathrm{~d}$ periodicity in the southern hemisphere that was reported by Antonucci et al. (1990) for cycle 21 was even more prominent during cycle 22. It has significantly diminished in cycle 23 . This periodicity is possibly related to the single hot spot system (i.e. a region with enhanced flare activity) that rotated with a constant period of $28.0 \mathrm{~d}$ as found by Bai (1990) for the southern hemisphere during cycles 19-22. In addition, we have shown that a (synodic) 25-25.5 d periodicity appeared in the southern hemisphere during all three cycles. This periodicity may be the photospheric signature of equatorially trapped Rossby waves with azimuthal order $m=1$. The northern hemisphere showed pronounced peaks at $26.9 \pm 0.1 \mathrm{~d}$ and its second harmonic $13.4 \pm 0.1 \mathrm{~d}$ during cycle 21, which was also noted by Antonucci et al. (1990). The $13.4 \mathrm{~d}$ periodicity can be understood in terms of a large-scale structures in the magnetic field such as two activity complexes separated by $180^{\circ}$ in longitude and rotating with the same period. We assume that the $26.9 \mathrm{~d}$ and $13.4 \mathrm{~d}$ periods are linked to a double-hot-spot system (two hot spots separated by $180^{\circ}$ ) that occurred during cycle 21 and had a rotation period of $26.7 \mathrm{~d}$ (Bai 2003a). The $26.9 \mathrm{~d}$ period agrees with the expected synodic rotation period of sunspots at these latitudes. During cycle 22, the northern hemisphere showed power at the slightly larger periodicities of 27.1-27.3 d. However, the dominant peak occurred at $29.0 \pm 0.1 \mathrm{~d}$ in the total flux and around 28.3-28.6 d in the net flux. During cycle 23, the dominant rotation period in the northern hemisphere was at $26.4 \pm 0.1 \mathrm{~d}$.

Although we have taken care to take into account as many significant quasi-periodicities as possible and to relate our results to previous studies, our coverage is not complete. In particular, we have omitted the periodicity range between $30 \mathrm{~d}$ and $100 \mathrm{~d}$. The reason is that power in this range drops to very low levels and it becomes increasingly difficult to distinguish possible signals from pure noise. More refined methods will be necessary to analyze this frequency interval. Nevertheless, we believe that our results provide a useful overview of the main quasi-periodic oscillations that have occurred in the large-scale photospheric magnetic field over the last three cycles 21-23. The present Fourier analysis of longitudinally averaged synoptic maps, originally introduced by Hoeksema \& Scherrer (1987) and Antonucci et al. (1990), has in combination with wavelet analysis proven to be a powerful tool for the detection of intrinsically solar periodicities in the photospheric magnetic field.

Acknowledgements. We thank P. A. Sturrock for constructive discussions. The NSO/Kitt Peak data used here were produced cooperatively by NSF/NOAO, NASA/GSFC, and NOAA/SEL. Wavelet software was provided by C. Torrence and G. Compo, and is available at URL: http://paos.colorado.edu/research/wavelets/. S. V. Berdyugina acknowledges support by the Academy of Finland, grant \#43039.

\section{References}

Antonucci, E., Hoeksema, J. T., \& Scherrer, P. H. 1990, ApJ, 360, 296

Atac, T., \& Ozguc, A. 1996, Sol. Phys., 166, 201

Bai, T. 1990, ApJ, 364, L17

Bai, T. 2003a, ApJ, 585, 1114

Bai, T. 2003b, ApJ, 591, 406 
Bai, T., \& Sturrock, P. A. 1991, Nature, 350, 141

Bai, T., \& Sturrock, P. A. 1993, ApJ, 409, 476

Ballester, J. L., Oliver, R., \& Carbonell, M. 2002, ApJ, 566, 505

Ballester, J. L., Oliver, R., \& Carbonell, M. 2004, ApJ, 615, L173

Balthasar, H., Vazquez, M., \& Woehl, H. 1986, A\&A, 155, 87

Bazilevskaya, G. A., Krainev, M. B., Makhmutov, V. S., et al. 2000, Sol. Phys., 197, 157

Benevolenskaya, E. E. 1998, ApJ, 509, L49

Berdyugina, S. V., \& Usoskin, I. G. 2003, A\&A, 405, 1121

Bogart, R. S. 1982, Sol. Phys., 76, 155

Bogart, R. S., \& Bai, T. 1985, ApJ, 299, L51

Brouwer, M. P., \& Zwaan, C. 1990, Sol. Phys., 129, 221

Bumba, V., \& Howard, R. 1969, Sol. Phys., 7, 28

Carbonell, M., \& Ballester, J. L. 1992, A\&A, 255, 350

Carbonell, M., Oliver, R., \& Ballester, J. L. 1993, A\&A, 274, 497

Chelton, D. B., \& Schlax, M. G. 1996, Science, 272, 234

De Moortel, I., Munday, S. A., \& Hood, A. W. 2004, Sol. Phys., 222, 203

Delache, P., Laclare, F., \& Sadsaoud, H. 1985, Nature, 317, 416

Duchlev, P. I. 2001, Sol. Phys., 199, 211

Duchlev, P. I., \& Dermendjiev, V. N. 1996, Sol. Phys., 168, 205

Dzhalilov, N. S., \& Staude, J. 2004, A\&A, 421, 305

Dzhalilov, N. S., Staude, J., \& Oraevsky, V. N. 2002, A\&A, 384, 282

Gaizauskas, V., Harvey, K. L., Harvey, J. W., \& Zwaan, C. 1983, ApJ, 265,1056

Gaizauskas, V., Mackay, D. H., \& Harvey, K. L. 2001, ApJ, 558, 888 Garcia, H. A. 1990, Sol. Phys., 127, 185

Gazis, P. R., Richardson, J. D., \& Paularena, K. I. 1995, Geophys. Res. Lett., 22, 1165

Goode, P. R., \& Thompson, M. J. 1992, ApJ, 395, 307

Gough, D. O., \& Kosovichev, A. G. 1992, in Inside the Stars, ed. W. W. Weiss, \& A. Baglin, Proc. IAU Coll., 137, 556

Gough, D. O., Kosovichev, A. G., \& Toutain, T. 1995, in GONG 1994, Helio- and Astro-Seismology from the Earth and Space, ASP Conf. Ser., 76, 55

Hansen, S. F., \& Hansen, R. T. 1975, Sol. Phys., 44, 503

Henney, C. J., \& Harvey, J. W. 2002, Sol. Phys., 207, 199

Hoeksema, J. T., \& Scherrer, P. H. 1987, ApJ, 318, 428

Howard, R. 1974, Sol. Phys., 38, 59

Howard, R., \& Labonte, B. J. 1981, Sol. Phys., 74, 131

Howe, R., Christensen-Dalsgaard, J., Hill, F., et al. 2000, Science, 287, 2456

Ichimoto, K., Kubota, J., Suzuki, M., Tohmura, I., \& Kurokawa, H. 1985, Nature, 316, 422

Javaraiah, J., \& Gokhale, M. H. 1997, Sol. Phys., 170, 389

Joshi, B., \& Joshi, A. 2004, Sol. Phys., 219, 343

Kiplinger, A. L., Dennis, B. R., \& Orwig, L. E. 1984, BAAS, 16, 891

Knaack, R., Stenflo, J. O., \& Berdyugina, S. V. 2004, A\&A, 418, L17

Krivova, N. A., \& Solanki, S. K. 2002, A\&A, 394, 701

Kuhn, J. R., Armstrong, J. D., Bush, R. I., \& Scherrer, P. 2000, Nature, 405, 544

Lean, J. 1990, ApJ, 363, 718

Lean, J. L., \& Brueckner, G. E. 1989, ApJ, 337, 568

Li, K.-J., Schmieder, B., \& Li, Q.-S. 1998, A\&AS, 131, 99

Li, K. J., Wang, J. X., Xiong, S. Y., et al. 2002, A\&A, 383, 648

Lockwood, M. 2001, J. Geophys. Res., 106, 16021

Lou, Y. 2000, ApJ, 540, 1102

Lou, Y., Wang, Y., Fan, Z., Wang, S., \& Wang, J. X. 2003, MNRAS, 345,809

Mouradian, Z., \& Soru-Escaut, I. 1991, A\&A, 251, 649

Mursula, K., Usoskin, I., \& Zieger, B. 1997, Sol. Phys., 176, 201
Mursula, K., Zieger, B., \& Vilppola, J. H. 2003, Sol. Phys., 212, 201

Neugebauer, M., Smith, E. J., Ruzmaikin, A., Feynman, J., \& Vaughan, A. H. 2000, J. Geophys. Res., 2315

Newton, H. W., \& Milsom, A. S. 1955, MNRAS, 115, 398

Obridko, V. N., \& Shelting, B. D. 2001, Astron. Rep., 45, 1012

Oliver, R., \& Ballester, J. L. 1994, Sol. Phys., 152, 481

Oliver, R., Ballester, J.-L., \& Baudin, F. 1998, Nature, 394, 552

Oliver, R., Carbonell, M., \& Ballester, J. L. 1992, Sol. Phys., 137, 141

Ossendrijver, M. 2003, A\&AR, 11, 287

Ozgüç, A., \& Ataç, T. 1996, Sol. Phys., 163, 183

Pap, J., Bouwer, S., \& Tobiska, W. 1990, Sol. Phys., 129, 165

Papaloizou, J., \& Pringle, J. E. 1978, MNRAS, 182, 423

Paularena, K. I., Szabo, A., \& Richardson, J. D. 1995, Geophys. Res. Lett., 22, 3001

Provost, J., Berthomieu, G., \& Rocca, A. 1981, A\&A, 94, 126

Rao, K. R. 1973, Sol. Phys., 29, 47

Reid, J. H. 1968, Sol. Phys., 5, 207

Richardson, J. D., Paularena, K. I., Belcher, J. W., \& Lazarus, A. J. 1994, Geophys. Res. Lett., 21, 1559

Rieger, E., Kanbach, G., Reppin, C., et al. 1984, Nature, 312, 623

Roy, J.-R. 1977, Sol. Phys., 52, 53

Ruzmaikin, A., Feynman, J., Neugebauer, M., \& Smith, E. J. 2001, J. Geophys. Res., 8363

Saio, H. 1982, ApJ, 256, 717

Schou, J., Antia, H. M., Basu, S., et al. 1998, ApJ, 505, 390

Silverman, S. M., \& Shapiro, R. 1983, J. Geophys. Res., 88, 6310

Smeyers, P., Craeynest, D., \& Martens, L. 1981, Ap\&SS, 78, 483

Snodgrass, H. B. 1984, Sol. Phys., 94, 13

Sokoloff, D., \& Nesme-Ribes, E. 1994, A\&A, 288, 293

Sturrock, P. A. 2004, ApJ, 605, 568

Sturrock, P. A., \& Bai, T. 1992, ApJ, 397, 337

Sturrock, P. A., Scargle, J. D., Walther, G., \& Wheatland, M. S. 1999, ApJ, 523, L177

Swinson, D., Koyama, H., \& Saito, T. 1986, Sol. Phys., 106, 35

Szabo, A., Lepping, R. P., \& King, J. H. 1995, Geophys. Res. Lett., 22,1845

Temmer, M., Veronig, A., \& Hanslmeier, A. 2002, A\&A, 390, 707

Temmer, M., Veronig, A., Hanslmeier, A., Otruba, W., \& Messerotti, M. 2001, A\&A, 375, 1049

Toomre, J., Christensen-Dalsgaard, J., Hill, F., et al. 2003, in ESA SP-517, 409

Torrence, C., \& Compo, G. P. 1998, Bull. Am. Met. Soc., 79, 61

Trotter, D. E., \& Billings, D. E. 1962, ApJ, 136, 1140

Ulrich, R. K. 2001, ApJ, 560, 466

Verma, V. K. 1987, Sol. Phys., 114, 185

Verma, V. K. 2000, A\&A, 21, 173

Vernova, E. S., Mursula, K., Tyasto, M. I., \& Baranov, D. G. 2002, Sol. Phys., 205, 371

Vizoso, G., \& Ballester, J. L. 1987, Sol. Phys., 112, 317

Vizoso, G., \& Ballester, J. L. 1989, Sol. Phys., 119, 411

Vizoso, G., \& Ballester, J. L. 1990, A\&A, 229, 540

Waldmeier, M. 1971, Sol. Phys., 20, 332

Wang, Y.-M., Nash, A. G., \& Sheeley, N. R. 1989, ApJ, 347, 529

Wang, Y.-M., \& Sheeley, N. R. 2003, ApJ, 590, 1111

Wolff, C. L. 1983, ApJ, 264, 667

Wolff, C. L. 1998, ApJ, 502, 961

Wolff, C. L. 2000, ApJ, 531, 591

Wolff, C. L., \& Blizard, J. B. 1986, Sol. Phys., 105, 1

Worden, J., \& Harvey, J. 2000, Sol. Phys., 195, 247

Zięba, S., Masłowski, J., Michalec, A., \& Kułak, A. 2001, A\&A, 377, 297 